MATHEMATICS OF COMPUTATION

Volume 72, Number 244, Pages 2013-2042

S 0025-5718(03)01503-5

Article electronically published on February 3, 2003

\title{
ON MINIMAL DISJOINT DEGENERATIONS FOR PREPROJECTIVE REPRESENTATIONS OF QUIVERS
}

\author{
KLAUS BONGARTZ AND THOMAS FRITZSCHE
}

\begin{abstract}
We derive a root test for degenerations as described in the title. In the case of Dynkin quivers this leads to a conceptual proof of the fact that the codimension of a minimal disjoint degeneration is always one. For Euclidean quivers, it enables us to show a periodic behaviour. This reduces the classification of all these degenerations to a finite problem that we have solved with the aid of a computer. It turns out that the codimensions are bounded by two. Somewhat surprisingly, the regular and preinjective modules play an essential role in our proofs.
\end{abstract}

\section{INTRODUCTION}

Throughout this article we are dealing with finite dimensional modules over the path algebra $k Q$ of a finite connected quiver without oriented cycles, i.e., with finite dimensional representations of $Q$. Thus the Auslander-Reiten quiver $\Gamma$ has a preprojective component containing all indecomposable projectives, and we call a module preprojective if its indecomposable direct summands all belong to that component. We denote by $U \preceq V$ the usual partial order between preprojective indecomposables, saying that there is a path from $U$ to $V$. The distance $d(U, V)$ between $U$ and $V$ is then the length of a shortest path leading from $U$ to $V$.

By [4, corollary 4.2], it is easy to see when a preprojective module $M$ degenerates to another preprojective $N$ of the same dimension vector. This happens to be true iff the inequality $\operatorname{dim} \operatorname{Hom}(M, W) \leq \operatorname{dim} \operatorname{Hom}(N, W)$ holds for all indecomposable preprojectives $W$ that are predecessors of some indecomposable direct summand of $M \oplus N$ iff there is a finite number of extensions $0 \rightarrow U_{i} \rightarrow M_{i} \rightarrow V_{i} \rightarrow 0$ with $M=M_{1}, M_{i+1}=U_{i} \oplus V_{i}$ and $N=U_{r} \oplus V_{r}$. We denote this by $M \leq N$. It follows from [5, theorem 4] that the minimal degenerations $M<N$ all come from a "minimal" extension $0 \rightarrow U \rightarrow M^{\prime} \rightarrow V \rightarrow 0$ with indecomposable ends by adding an appropriate common direct summand $X$, and by setting $M=M^{\prime} \oplus X$ and $N=U \oplus V \oplus X$. Here, $M^{\prime}$ and $U \oplus V$ are disjoint, i.e., they have no indecomposable direct summand in common. However, the minimal disjoint degenerations induced by $0 \rightarrow U \rightarrow M^{\prime} \rightarrow V \rightarrow 0$, and interesting invariants thereof like the codimension of the orbit closures, are not known in general even though for given $U$ and $V$ a computer can easily determine all possible $M^{\prime}$, at least in principle. Thus for Dynkin quivers, using the shrinking of bijectively represented arrows that reduces the infinite families $A_{n}, D_{m}$ to the cases $n \leq 3, m \leq 6$, one ends up with a finite list that has already been determined with the help of a computer in [10]. For a

Received by the editor March 1, 2001 and, in revised form, March 4, 2002.

2000 Mathematics Subject Classification. Primary 16G20, 14L30. 
Euclidean (or tame or extended Dynkin) quiver of type $\tilde{A}_{n}, \tilde{D}_{m}, \tilde{E}_{6}, \tilde{E}_{7}$ or $\tilde{E}_{8}$ it is not so obvious that the classification is a finite problem that can be solved with a computer. We now want to explain how and in which sense this can be achieved.

First of all, one can always tilt to the case where $U$ is the only simple projective. From now on this will be taken for granted. By shrinking some appropriate arrows one reduces further to the finite list of quivers of type $\tilde{A}_{n}, \tilde{D}_{m}, \tilde{E}_{6}, \tilde{E}_{7}$ or $\tilde{E}_{8}$ with $n \leq 3, m \leq 8$. These quivers have finite diameter $d \leq 7$ and Coxeter numbers $p \leq 30$, i.e., $p$ is the order of the corresponding Coxeter-transformation modulo the space generated by the null-root. Now, as soon as $d(U, V) \geq 4(p+d)$, the following holds for any minimal degeneration $M<U \oplus V: M$ can be decomposed into $M_{1} \oplus M_{2}$ such that all indecomposable direct summands $W$ of $M_{1}$ satisfy $d(U, W)<2(p+d)$ and dually all direct summands of $M_{2}$ satisfy $d(W, V)<2(p+d)$. Furthermore, $M_{1} \oplus \operatorname{Tr} D^{p} M_{2}$ has $U \oplus \operatorname{Tr} D^{p} V$ as a minimal degeneration, and any minimal degeneration ending there is of this form. This correspondence preserves the codimensions. However, finer invariants like the type of singularities are not even preserved in the simple case of the double arrow (see [3]).

Since we only have to look at the finitely many modules $V$ reachable from the finitely many $U$ by paths with at most $6 p+4 d$ arrows the enumeration of all minimal disjoint degenerations is now a finite problem. In particular, there exist natural numbers $n$ and $c$ such that for any pair $U, V$ of indecomposable preprojective representations over any tame quiver one has at most $n$ minimal degenerations $M<U \oplus V$ and the codimension of such a degeneration is always at most $c$. In fact, the best values for $n$, resp. $c$, are 613 , resp. 2, as found with the help of a computer. For some more details on the numerical aspects we refer to the tables at the end of this article. Full details and the source-code of the programs can be found at http://wmaz.math.uni-wuppertal.de by clicking first at the link "activities", then at "minimal degenerations of extended Dynkin quivers", then at "Web-Interface" and by following the instructions given there.

The above reduction and an algorithm fast enough to carry through the classification on a personal computer are both based on the following root test for minimal degenerations that is valid for arbitrary quivers. Let $0 \rightarrow U \rightarrow M=M_{1} \oplus M_{2} \rightarrow$ $V \rightarrow 0$ induce a minimal degeneration. Suppose that the decomposition of $M$ is directed, i.e., that no direct summand of $M_{2}$ is a proper predecessor of a direct summand of $M_{1}$. Then for $M_{1} \neq 0$ the right end term in the induced exact sequence $0 \rightarrow U \rightarrow M_{1} \rightarrow R \rightarrow 0$ is indecomposable. It follows in particular that the function $W \mapsto \operatorname{dim} \operatorname{Hom}(U \oplus V, W)-\operatorname{dim} \operatorname{Hom}(M, W)$ is a root or 0 when restricted to any slice.

We use the root test also for a conceptual proof of the known fact that the codimensions of minimal disjoint degenerations are always one for representations of Dynkin quivers.

The problem dealt with in this article was posed by the first author to the second author as a computer-aided "Diplomarbeit". However, the more or less evident algorithms used before the root-test was available were too slow to ever reach the large bounds the first author had at that time for type $\tilde{E}_{8}$. He was led to the root test by inspection of the numerical results the second author had obtained so far. Thus the dialectical process between theory and experiment was useful for both sides (and for both authors). 
Throughout this article, the following notations and assumptions are valid. We work over an algebraically closed field $k$ of arbitrary characteristic. All our representations and modules will be of finite dimension over $k$. Basic notions from representation theory are used without any further comments; in particular, almost split sequences, Auslander-Reiten quivers and the "functors" DTr and TrD. Note that for hereditary algebras like path algebras, $D T r$, resp. $\operatorname{Tr} D$, are indeed left, resp. right, exact functors. The abbreviations $[X, Y]$ and $[X, Y]^{1}$ denote the dimensions of $\operatorname{Hom}(X, Y)$ and $\operatorname{Ext}^{1}(X, Y)$. For two modules $M, N$ with the same dimension vector $\underline{\operatorname{dim}} M=\underline{\operatorname{dim}} N$, one has for all indecomposables $T$ the nice formula

$$
[M, T]-[N, T]=[\operatorname{Tr} D T, M]-[\operatorname{Tr} D T, N]
$$

(see [2, cor. 4.3]). Also the famous formula

$$
D \underline{\operatorname{Hom}}(X, Y) \simeq \operatorname{Ext}^{1}(Y, D T r X)
$$

(see e.g., [2, prop. 4.5]), valid for all modules, is used several times.

\section{The ROOT TEST FOR MINIMAL DISJOINT DEGENERATIONS}

2.1. The main observation. If $M<U \oplus V$ is a degeneration with indecomposable preprojective modules $U$ and $V$ over an arbitrary path algebra $k Q$ that satisfy $U \prec V$, then there is by [5, theorem 4.5] an injection $\varepsilon: U \rightarrow M$ with cokernel $V$. Now choose any directed decomposition $M=M_{1} \oplus M_{2}$ of $M$ with $M_{1} \neq 0 \neq M_{2}$ and decompose $\varepsilon$ into the corresponding components $\varepsilon_{1}$ and $\varepsilon_{2}$.

Theorem 1. Keep all the above notations and assumptions. Suppose furthermore that $M<U \oplus V$ is a minimal degeneration. Then the cokernel of $\varepsilon_{1}$ is indecomposable.

Proof. First of all, $\varepsilon_{1}$ is not 0 , and so it is injective ( $U$ is simple!), because otherwise $\varepsilon$ splits. So we start with the following exact commutative diagram:

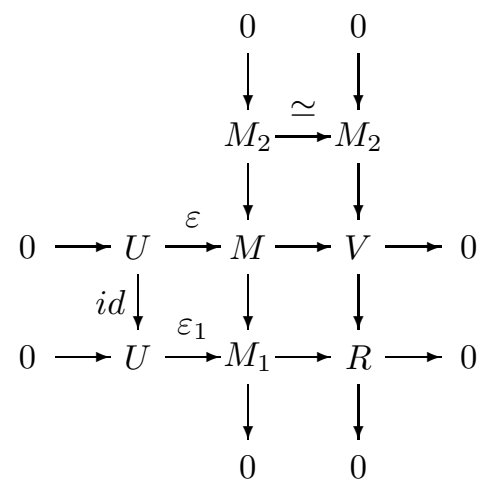

Assume now that $R=R_{1} \oplus R_{2}$ holds with an indecomposable $R_{1}$. We have to show that $R_{2}$ vanishes. The inclusion of $R_{1}$ and the projection onto $R_{1}$ induce the following two commutative exact diagrams: 

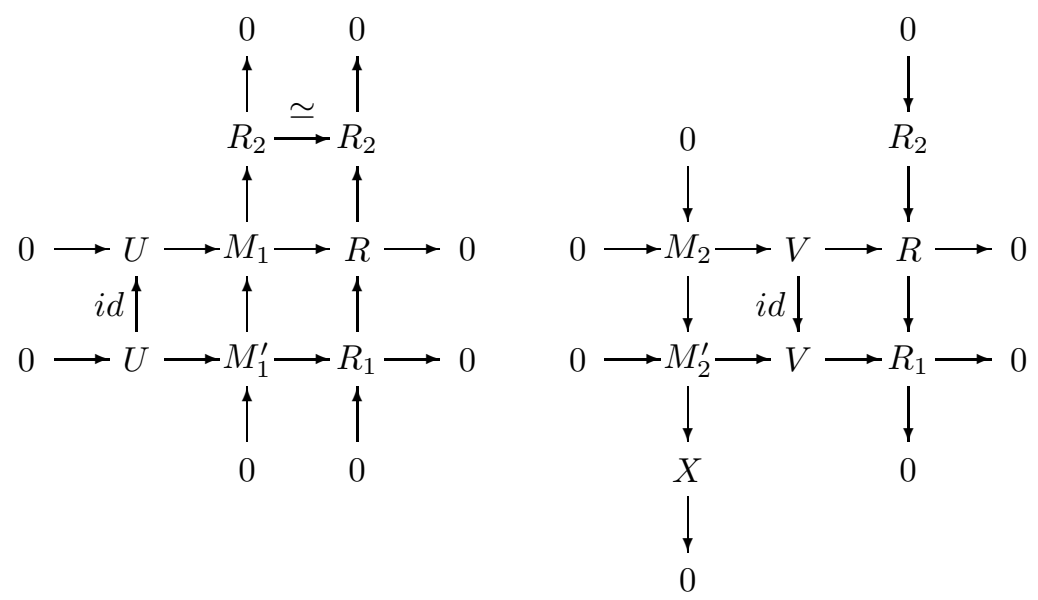

By the snake lemma, $X$ is isomorphic to $R_{2}$. We extract the following four short exact sequences:

$$
\begin{aligned}
& (1): 0 \rightarrow U \rightarrow M_{1}^{\prime} \rightarrow R_{1} \rightarrow 0, \\
& (2): 0 \rightarrow M_{1}^{\prime} \rightarrow M_{1} \rightarrow R_{2} \rightarrow 0, \\
& (3): 0 \rightarrow M_{2}^{\prime} \rightarrow V \rightarrow R_{1} \rightarrow 0, \\
& (4): 0 \rightarrow M_{2} \rightarrow M_{2}^{\prime} \rightarrow R_{2} \rightarrow 0 .
\end{aligned}
$$

Our claim is that $M \leq M^{\prime}=M_{1}^{\prime} \oplus M_{2}^{\prime} \leq U \oplus V$. Thus let $T \prec V$ be a preprojective indecomposable. Because $[R, T]=0$, we obtain induced exact sequences

$$
\begin{aligned}
& (1 *): 0 \rightarrow \operatorname{Hom}\left(M_{1}^{\prime}, T\right) \rightarrow \operatorname{Hom}(U, T) \rightarrow \operatorname{Ext}\left(R_{1}, T\right) \rightarrow \operatorname{Ext}\left(M_{1}^{\prime}, T\right), \\
& (2 *): 0 \rightarrow \operatorname{Hom}\left(M_{1}, T\right) \rightarrow \operatorname{Hom}\left(M_{1}^{\prime}, T\right) \rightarrow \operatorname{Ext}\left(R_{2}, T\right) \rightarrow \operatorname{Ext}\left(M_{1}, T\right), \\
& (3 *): 0 \rightarrow \operatorname{Hom}(V, T) \rightarrow \operatorname{Hom}\left(M_{2}^{\prime}, T\right) \rightarrow \operatorname{Ext}\left(R_{1}, T\right) \rightarrow \operatorname{Ext}(V, T), \\
& (4 *): 0 \rightarrow \operatorname{Hom}\left(M_{2}^{\prime}, T\right) \rightarrow \operatorname{Hom}\left(M_{2}, T\right) \rightarrow \operatorname{Ext}\left(R_{2}, T\right) \rightarrow \operatorname{Ext}\left(M_{2}^{\prime}, T\right) .
\end{aligned}
$$

First suppose that $\left[M_{2}, T\right]=0$. By $(4 *)$ we also have $\left[M_{2}^{\prime}, T\right]=0$. Using $(2 *)$ and $(1 *)$, we find that

$$
\left[M_{1} \oplus M_{2}, T\right]=\left[M_{1}, T\right] \leq\left[M_{1}^{\prime}, T\right]=\left[M_{1}^{\prime} \oplus M_{2}^{\prime}, T\right] \leq[U, T] \leq[U \oplus V, T] .
$$

Now assume that $\left[M_{2}, T\right] \neq 0$. Then we have $0=\left[M_{1}, T\right]^{1}=\left[\operatorname{Tr} D T, M_{1}\right]$, because the decomposition of $M$ is directed. We also have $0=\left[M_{1}^{\prime}, T\right]^{1}$ for the submodule $M_{1}^{\prime}$ of $M_{1}$. Using $(2 *),(4 *),(1 *)$ and $(3 *)$ in this order, we obtain

$$
\begin{aligned}
& {\left[M_{1} \oplus M_{2}, T\right] \leq\left[M_{1}^{\prime}, T\right]-\left[R_{2}, T\right]^{1}+\left[M_{2}^{\prime}, T\right]+\left[R_{2}, T\right]^{1}} \\
& \quad=\left[M_{1}^{\prime} \oplus M_{2}^{\prime}, T\right] \leq[U, T]-\left[R_{1}, T\right]^{1}+[V, T]+\left[R_{1}, T\right]^{1}=[U \oplus V, T] .
\end{aligned}
$$

Since $M, M^{\prime}$ and $U \oplus V$ all have the same dimension vector and contain only predecessors of $V$ as direct summands, we get for all indecomposable modules $W$ that are not preprojective or not $\preceq$-smaller than $D \operatorname{Tr} V$ the equalities

$$
[U \oplus V, W]-[M, W]=[\operatorname{Tr} D W, U \oplus V]-[\operatorname{Tr} D W, M]=0
$$

and

$$
[U \oplus V, W]-\left[M^{\prime}, W\right]=[\operatorname{Tr} D W, U \oplus V]-\left[\operatorname{Tr} D W, M^{\prime}\right]=0 .
$$

Thus we have proved our claim $M \leq M^{\prime} \leq U \oplus V$. 
If $M^{\prime}$ is isomorphic to $U \oplus V$, we have $M_{1}^{\prime} \simeq U$, whence the contradiction $R_{1}=0$. So we have $M \simeq M^{\prime}$ by minimality, and it remains to be seen that $R_{2}=0$.

If not, we have $\operatorname{dim} M_{2}<\operatorname{dim} M_{2}^{\prime}$. From the exact sequence

$$
0 \rightarrow M_{2} \rightarrow M_{2}^{\prime} \rightarrow R_{2} \rightarrow 0
$$

we split off an exact sequence of type

$$
0 \rightarrow Z \rightarrow Z \rightarrow 0 \rightarrow 0
$$

with $Z$ of maximal dimension, and we denote the remaining sequence again as in (4). By the Krull-Remak-Schmidt theorem there is an indecomposable direct summand $X$ in $M_{2}^{\prime}$ also occuring in $M_{1}$, and we fix such an $X$. We write $M_{2}$ as a direct sum of indecomposables $Y_{1}, Y_{2}, \ldots Y_{r}$, and we look at the homomorphisms $\alpha(i): Y(i) \rightarrow X$ induced by the injection $M_{2} \rightarrow M_{2}^{\prime}$ occurring in (4). If we have $\alpha(i)=0$ for all $i$, then $X$ is a direct summand of $R_{2}$ because of the exact sequence (4). So we obtain $X \prec V \preceq X$, a contradiction. Thus we have $\alpha(i) \neq 0$ for at least one index $i$. By the definition of a directed decomposition, $Y_{i} \prec X$ is not possible. Thus we have $Y_{i}=X$. Then $\alpha(i)$ is invertible, and we can split off

$$
0 \rightarrow X \rightarrow X \rightarrow 0 \rightarrow 0
$$

once more, in contradiction to the choice of $Z$.

Remark 1. Of course, the isomorphism class of the cokernel $R$ depends heavily on the choice of $\varepsilon$ and the decomposition.

2.2. The root test. Let $S$ be a slice in the preprojective component of $k Q$ as introduced by Ringel in [13, page 180]. Thus for any point $x$ in the quiver with corresponding indecomposable projective $P(x)$ there is exactly one index $n(x)$ such that $X=\operatorname{Tr} D^{n(x)} P(x)$ belongs to $S$. For any minimal disjoint degeneration $M<$ $U \oplus V$ with directed decomposition $M=M_{1} \oplus M_{2}$ we look at the vector $s$ in $\mathbf{N}^{Q_{0}}$ defined by

$$
s(x)=[U, X]-\left[M_{1}, X\right] .
$$

We claim that this vector or its negative is always a root of $Q$, i.e., the dimension vector of an indecomposable. By a deep result of Kac (9]) this notion coincides with the usual definition in Lie theory. In particular, it is independent of the orientation of the quiver. For Dynkin quivers and Euclidean quivers the situation is much easier, because the roots are precisely the nonnegative integer vectors where the quadratic form attached to the quiver takes values 1 or 0 .

Lemma 1. Using the above notation, we have:

a) The vector $s$ or its negative is a root of $Q$. If $V$ is not a predecessor of some module in $S$, it is a root.

b) The restriction of the function $W \mapsto[U \oplus V, W]-[M, W]$ to $S$ is a root or 0 .

Proof. a) Of course, we can assume that each direct summand of $M_{1}$ is a predecessor of some module in $S$, because the others do not contribute to $s$. The direct sum $T$ of all the modules in $S$ is a tilting module whose endomorphism algebra is again the path algebra of a quiver with the same underlying graph as $Q$. We distinguish several possibilities. 
For $M_{1}=0$ we have $s(x)=[U, X]$, whence dualization and tilting show that $s$ is a dimension vector. If $M_{1}$ does not vanish, we look at an exact sequence

$$
0 \rightarrow U \rightarrow M_{1} \rightarrow R \rightarrow 0
$$

with indecomposable end as in theorem 1. For any $X$ in $S$ we obtain an induced exact sequence

$$
0 \rightarrow \operatorname{Hom}(R, X) \rightarrow \operatorname{Hom}\left(M_{1}, X\right) \rightarrow \operatorname{Hom}(U, X) \rightarrow \operatorname{Ext}(R, X) \rightarrow 0 .
$$

Since $T$ is a tilting module and $R$ is not 0 , we cannot have $[R, X]=0=[R, X]^{1}$ for all $X$ in $S$. Now suppose for a moment that we have $[R, X] \neq 0 \neq\left[R, X^{\prime}\right]^{1}$ for some $X, X^{\prime}$ in $S$. Then we get $X^{\prime} \preceq \operatorname{Tr} D X^{\prime} \preceq R \preceq X$, so that $X^{\prime}$ and $\operatorname{Tr} D X^{\prime}$ belong to the slice $S$, which is path closed. This contradiction shows that there are only two possibilities left.

Either we have

$$
s(x)=[U, X]-\left[M_{1}, X\right]=[R, X]^{1}=[X, D T r R]
$$

for all $x$, or else

$$
s(x)=[U, X]-\left[M_{1}, X\right]=-[R, X] .
$$

In the first case, $D \operatorname{Tr} R$ is generated by $T$ as a successor of a slice module. So our claim follows directly from tilting theory. In the second case we dualize first and apply tilting afterwards.

If $V$ is not a predecessor of some $X$ in $S$, then also $R$ is not a predecessor. This implies $[R, X]=0$ for all $X$, and excludes the second possibility above.

b) Let $M_{1}$ be the direct sum of all indecomposable summands with multiplicities of $M$ that are predecessor of some module on $S$, and let $M_{2}$ be the remaining part. If $V$ is not a predecessor of some module on $S$, we can apply part a) and obtain a root. If $V$ is a predecessor, we have $M=M_{1}$ and $R=V$. Then our vector is 0 .

2.3. An application to Dynkin quivers. Here we consider a Dynkin quiver $Q$ with associated positive definite quadratic form $q$ and corresponding symmetric bilinear form $b$. The next result has been shown in [5, 10] by a tedious calculation.

Lemma 2. A minimal disjoint degeneration between representations of a Dynkin quiver has codimension one.

Proof. If not, there is by [5, lemma 4] a minimal degeneration induced by

$$
0 \rightarrow U \rightarrow X^{2} \oplus Y \rightarrow V \rightarrow 0
$$

for some indecomposable $X$. Then no sectional path with start $X$ ends up in a projective $P$ because of $2 \leq\left[X^{2} \oplus Y, P\right] \leq[U \oplus V, P]$. Thus there is a slice $S$ containing $X$ as a sink. Then we take as $M_{1}$ all indecomposable summands of $M$ (counted with multiplicities) that are predecessors of some module in $S$. In $M_{1}^{\prime}$ we remove two copies of $X$. For the corresponding vectors $s$ and $s^{\prime}$ we have $s^{\prime}=s+2 e$ with the canonical base vector $e$ to the point $x$. The relations

$$
1=b\left(s^{\prime}, s^{\prime}\right)=b(s, s)+4 b(s, e)+4 b(e, e)
$$

and

$$
0<b(s+e, s+e)=b(s, s)+2 b(s, e)+b(e, e),
$$

give us the desired contradiction. 


\section{TAME QUIVERS}

3.1. Some known results and the shrinking of an arrow. We recall some well-known facts that the reader can find in [7, 13]. For tame quivers, an indecomposable representation is either preprojective or else preinjective or else regular. Accordingly, any module $M$ can be decomposed in an essentially unique way into its preprojective part $M_{P}$, its regular part $M_{R}$ and its preinjective part $M_{I}$. An important fact is that there are no nonzero maps from preinjectives to regular or preprojective modules and from regular modules to preprojectives. The full subcategory $\mathcal{R}$ of all regular modules is an abelian subcategory which breaks up into a direct sum of subcategories $\mathcal{T}(p), p \varepsilon \mathbf{P}^{1}(k)$. Each of these categories is equivalent to the category $\mathcal{N}\left(n_{p}\right)$ of nilpotent representations of an oriented cycle with $n_{p}$ points. In fact, for all but at most three values of $p$ we have $n_{p}=1$, i.e., $\mathcal{T}(p)$ has only one simple object; and these categories are called homogeneous. In the other cases the simples are conjugate under $D T r$.

The tame quivers are characterized by the fact that the associated quadratic form

$$
q(x)=\sum_{i \varepsilon Q_{0}} x_{i}^{2}-\sum_{i \Sigma_{j}} x_{i} x_{j}
$$

is positive semi-definite on $\mathbf{R}^{Q_{0}}$. Its radical admits a generator $\underline{n}$ in $\mathbf{N}^{Q_{0}}$ with strictly positive entries, one of which is 1 . The sum of the dimension vectors of all simple regular modules in a category $\mathcal{T}(p)$ as above equals $\underline{n}$. The global dimension of a quiver algebra is at most one, so that the map $(M, N) \mapsto[M, N]-[M, N]^{1}$ induces a bilinear form on the Grothendieck group $K_{0}(A)$. Identifying $K_{0}(A)$ and $\mathbf{Z}^{Q_{0}}$ by $\underline{\text { dim }}$, we see that its associated quadratic form is $q$.

There is a linear form $\partial$ on $K_{0}(A)$, called the defect, whose values on the dimension vector of an indecomposable are strictly negative for a preprojective, zero for a regular and strictly positive for a preinjective. Given any module $E$ with dimension vector $\underline{n}$, one has $\partial(\underline{\operatorname{dim}} U)=[U, E]^{1}-[U, E]$. Quite often we simply write $\partial U$ instead of $\partial(\underline{\operatorname{dim}} U)$. Another definition of the defect uses the Coxeter transformation $c$. This is the unique endomorphism of $\mathbf{R}^{Q_{0}}$ that sends $\underline{\operatorname{dim}} U$ to $\underline{\operatorname{dim}} D \operatorname{Tr} U$ for each nonprojective indecomposable $U$. In particular, $\underline{n}$ is fixed by $c$, which induces on the quotient space an automorphism of finite order $p=p(Q)$. Then there is for any indecomposable $U$ the formula

$$
c^{p}(\underline{\operatorname{dim}} U)=\underline{\operatorname{dim}} U+\varepsilon(Q) \partial(\underline{\operatorname{dim}} U) \underline{n},
$$

where $\varepsilon(Q)$ is a strictly positive natural number depending on $Q$.

To reduce the infinite families of quivers of types $\tilde{A}_{n}$ and $\tilde{D}_{m}$ to the cases $n \leq 3$ and $m \leq 8$ we apply the known technique of shrinking an arrow to a point. Thus we look at some arrow $\varphi: x \rightarrow y$ in $Q$. Then we obtain the shrunken quiver $Q^{\prime}$ by identifying the two points $x$ and $y$ and by forgetting $\varphi$. Clearly, the full subcategory $C$ of all representations $M$ of $Q$ with bijective $M(\varphi)$ is under the obvious functor $M \mapsto M^{\prime}$ equivalent to the category of all representations of $Q^{\prime}$. If $N$ belongs to $C$, then all degenerations $M$ to $N$ also do, because bijectivity is an open condition. Furthermore, $M \mapsto M^{\prime}$ induces a bijection between the set of all minimal degenerations to $N$ and the set of all minimal degenerations to $N^{\prime}$. This bijection respects codimensions of orbits, decompositions into indecomposables, and even finer invariants like types of singularities occurring in orbit closures. 
We want to show that this reduction applies to the direct sum $U \oplus V$ of two indecomposable preprojectives over a path algebra of type $\tilde{A}_{n}$ or $\tilde{D}_{m}$ as soon as $n \geq 4$ or $m \geq 9$. By our general convention, $U$ is the projective simple $P(\omega)$ to the only sink $\omega$ in $Q$. To find an arrow $\varphi: x \rightarrow y$ represented by a bijection in $U$ and $V$ we only have to check whether $\operatorname{dim} V(x)=\operatorname{dim} V(y)$ holds for some arrow not ending in $\omega$ (see [13] page 76]). Then the quiver $Q^{\prime}$ again contains no oriented cycle, and $V^{\prime}$ is still preprojective.

Now, by a result of Moody in [11] or [7, lemma 1.8], the dimension vector $\underline{\operatorname{dim} V}$ of a preprojective indecomposable can always be written as $\underline{\operatorname{dim}} V=n \underline{n}+\underline{v}^{\prime}$ for some appropriate natural number $n$ and some root $\underline{v}^{\prime} \neq \underline{n}$ with $\underline{v}^{\prime} \leq \underline{n}$. Here $\underline{v}^{\prime} \leq \underline{n}$ means $\underline{v^{\prime}}(i) \leq \underline{n}(i)$ for all points $i$ in the quiver.

First, we look at a quiver of type $\tilde{A}_{n}$. Let $\alpha$ be the only source in $Q$. Then we have $\underline{v}^{\prime}(\alpha)=0$, because $\underline{v}^{\prime}$ is the dimension vector of a preprojective indecomposable. If there is a path from $\alpha$ to $\omega$ with at least three arrows, one of these is represented by a bijection in $U$ and $V$, so that we can reduce to $Q^{\prime}$, which is of type $\tilde{A}_{n-1}$. Thus we can reduce to the case $n \leq 3$ with at most two composable arrows.

Similarly, for a quiver of type $\tilde{D}_{m}$ we look at the $m-4$ arrows between the two branching points. In a preprojective indecomposable, at most two of them are not represented by a bijection. Thus we can always reduce for $m \geq 9$.

3.2. A sharpening of the root test. For tame quivers, theorem 1 can be refined as follows:

Lemma 3. Suppose we have a minimal degeneration $M<U \oplus V$ of preprojectives and a directed decomposition $M=M_{1} \oplus M_{2}$ such that the defects of $U$ and $M_{1}$ coincide. Then we have $\underline{\operatorname{dim} R} \leq \underline{n}$ in theorem 1 .

Proof. By the additivity of the defect in short exact sequences, $R$ is a regular indecomposable module. If $\underline{\operatorname{dim} R} \leq \underline{n}$ is not true, we find short exact sequences of regular modules of the form

$$
0 \rightarrow R_{1} \rightarrow R \rightarrow R_{2} \rightarrow 0
$$

and

$$
0 \rightarrow R_{1}^{\prime} \rightarrow R \rightarrow R_{2}^{\prime} \rightarrow 0
$$

with $\underline{\operatorname{dim}} R_{1}=\underline{n}=\underline{\operatorname{dim}} R_{1}^{\prime}$. Namely, $R_{1}$, resp. $R_{1}^{\prime}$, is the uniquely determined regular uniserial submodule, resp. factor module, of $R$ that contains each simple belonging to the tube of $R$ exactly once as a regular composition factor. As in the proof of theorem 1 , we consider the two diagrams induced from the exact sequence

$$
0 \rightarrow U \rightarrow M_{1} \rightarrow R \rightarrow 0
$$

by the inclusion $R_{1} \rightarrow R$ and from the exact sequence

$$
0 \rightarrow M_{1} \rightarrow V \rightarrow R \rightarrow 0
$$

by the projection $R \rightarrow R_{1}^{\prime}$. Then we obtain the four exact sequences

$$
\begin{aligned}
& (1): 0 \rightarrow U \rightarrow M_{1}^{\prime} \rightarrow R_{1} \rightarrow 0, \\
& (2): 0 \rightarrow M_{1}^{\prime} \rightarrow M_{1} \rightarrow R_{2} \rightarrow 0, \\
& (3): 0 \rightarrow M_{2}^{\prime} \rightarrow V \rightarrow R_{1}^{\prime} \rightarrow 0, \\
& (4): 0 \rightarrow M_{2} \rightarrow M_{2}^{\prime} \rightarrow R_{2}^{\prime} \rightarrow 0 .
\end{aligned}
$$


Since the dimension vectors of the dashed and undashed regular modules $R_{i}$ and $R_{i}^{\prime}$ coincide, we find for all indecomposable preprojectives $T$ the equalities

$$
\left[R_{i}, T\right]^{1}=\left[R_{i}, T\right]^{1}-\left[R_{i}, T\right]=\left[R_{i}^{\prime}, T\right]^{1}-\left[R_{i}^{\prime}, T\right]=\left[R_{i}^{\prime}, T\right]^{1} .
$$

Arguing as in the proof of theorem 1, we construct a module $M^{\prime}=M_{1}^{\prime} \oplus M_{2}^{\prime}$ such that $M \leq M^{\prime} \leq U \oplus V$. Again, $M^{\prime}$ cannot be isomorphic to $U \oplus V$. But this time, it cannot be isomorphic to $M$ either, because $R_{2} \neq 0$. This contradiction to the minimality shows that $\underline{\operatorname{dim} R} \leq \underline{n}$ is true.

3.3. Direct summands of defect $\leq-2$ are not far away from the ends. In the following lemma we have not tried to give the sharpest bounds that are possible, because this would make the arguments much more involved.

Lemma 4. Let $M<U \oplus V$ be a minimal disjoint degeneration with directed decomposition $M=M_{1} \oplus M_{2}$ satisfying $\partial M_{1}>\partial U$. Assume that $X$ is a $\preceq$-minimal direct summand in $M_{2}$ with $d(U, X) \geq 2(p+d)$. Then $\partial X=-1$.

Proof. Suppose not. Unfortunately, we have to consider various cases, so our proof is a bit technical. However, the reasoning runs always as follows:

We start with a $\preceq$-minimal direct summand $X$ in $M_{2}$ with $d(U, X) \geq 2(p+d)$ and $\partial X<-1$. Applying the general strategy of [1, 12, we construct another possibly trivial or decomposable direct summand $X^{\prime}$ of $M=M^{\prime} \oplus X \oplus X^{\prime}$ and an obvious degeneration $Z$ of $X \oplus X^{\prime}$ such that $M<M^{\prime} \oplus Z<U \oplus V$. To show the last inequality, we verify with the help of the root test that for all preprojective indecomposables $W$ we have

$$
\delta(W):=[Z, W]-\left[X \oplus X^{\prime}, W\right] \leq \varepsilon(W):=[U \oplus V, W]-[M, W] .
$$

Here $\delta(W)$ is always at most one, so we only have to check that the support of $\delta$ is contained in the support of $\varepsilon$.

First, we treat the difficult cases $\tilde{E}_{6}, \tilde{E}_{7}$ and $\tilde{E}_{8}$, using the following notation with $r \geq s \geq t \geq 1$ :

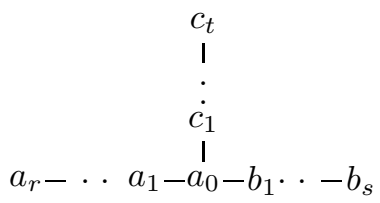

By our assumption $d(U, X) \geq 2(p+d)$ the slice $S$ through $X$ with only one source $A_{r}$ lying in the $\operatorname{Tr} D$-orbit of $P\left(a_{r}\right)$ exists in the Auslander-Reiten quiver. Observe that the defect of $A_{r}$ is always -1 , so that $X$ is different from $A_{r}$. For all points $y$ in the quiver, the module in $S$ belonging to the $\operatorname{Tr} D$-orbit of $P(y)$ is simply denoted by $Y$. Part b) of lemma 1 says that the restriction of $\varepsilon$ to $S$ is a root or 0 . We claim that $\varepsilon(Y) \geq \underline{n}(y)$ holds for the only predecessor $Y$ of $X$ in $S$. Namely, one has

$$
\varepsilon(Y)=[U, Y]-\left[M_{1}, Y\right]
$$

because $X$ is a $\preceq$-minimal direct summand of $M_{2}$. Since $d(U, X) \geq 2(p+d)$, $Y$ equals $\operatorname{Tr} D^{b} P(y)$ for some point $y$ and some $b \geq p$. Thus our claim follows immediately in the case $M_{1}=0$. In the other case we consider the exact sequence

$$
0 \rightarrow U \rightarrow M_{1} \rightarrow R \rightarrow 0
$$


given by theorem 1. By our assumptions on the defects, $R$ equals $D T r^{a} I$ for some injective indecomposable $I$. Applying $\operatorname{Hom}(, Y)$ to the above sequence gives

$$
[U, Y]-\left[M_{1}, Y\right]=[R, Y]^{1}=[\operatorname{Tr} D Y, R]=\left[P(y), D \operatorname{Tr}^{a+b+1} I\right] \geq \underline{n}(y) .
$$

A similar argument will be used in the sequel quite often for modules other than $Y$.

Now we study the different possibilities for $X$. We start with the simple case that $X=A_{i}$ for some $i<r$. If we have $\varepsilon\left(A_{j}\right)>0$ for all $j>i$, we set $X^{\prime}=0$, $Z=A_{r} \oplus \operatorname{Tr} D A_{i+1}$, and obtain $M<M^{\prime} \oplus Z<U \oplus V$. If $\varepsilon\left(A_{j}\right)=0$ for some $j>i$, we get from $\varepsilon\left(A_{i+1}\right) \geq \underline{n}\left(a_{i+1}\right)$, the shape of the null root $\underline{n}$ and the tables of the roots for Dynkin quivers in [6] that $\varepsilon$ vanishes only at $A_{r}$. Because $D \operatorname{Tr}^{p} A_{r}$ exists, we have $\left[U, A_{r}\right] \neq 0$, whence $M_{1} \neq 0$, and an application of $\operatorname{Hom}\left(, A_{r}\right)$ to the exact sequence above shows that

$$
\begin{aligned}
\varepsilon\left(A_{r}\right) & =\left[U, A_{r}\right]-\left[M_{1}, A_{r}\right]=\left[R, A_{r}\right]^{1}-\left[M_{1}, A_{r}\right]^{1} \\
& =\left[\operatorname{Tr} D A_{r}, R\right]-\left[\operatorname{Tr} D A_{r}, M_{1}\right]=0 .
\end{aligned}
$$

Thus $\left[\operatorname{Tr} D A_{r}, M_{1}\right]=\left[\operatorname{Tr} D A_{r}, R\right]=\left[P\left(a_{r}\right), D \operatorname{Tr}^{m} I\right] \neq 0$ holds, because $m \geq p$. Therefore $M_{1}$ contains some $\operatorname{Tr} D A_{j}$ as a direct summand with $\operatorname{Tr} D A_{j} \npreceq X$. We take the smallest index $j$ with this property, and set $X^{\prime}=\operatorname{Tr} D A_{j}$ and $Z=A_{j-1} \oplus$ $\operatorname{Tr} D A_{i+1}$.

Observe that by symmetry the proof is already complete for the type $\tilde{E}_{6}$, and for $\tilde{E}_{7}$ only the case $X=C_{1}$ remains to be done. If $S$ lies in the support of $\varepsilon$, we can take $X^{\prime}=0$ and $Z=A_{3} \oplus \operatorname{Tr} D B_{3}$. There remain only two possibilities for the vanishing of $\varepsilon$ on $S$, because we have $\varepsilon\left(A_{0}\right) \geq 4$ and because the restriction to $S$ is a root (see again the tables in [6]). Either we have $\varepsilon\left(A_{3}\right)=0$, or else $\varepsilon\left(B_{3}\right)=0$. In the first case, there is (as in the case $X=A_{i}$ treated before) a smallest index $j>0$ such that $\operatorname{Tr} D A_{j}$ is a direct summand of $M_{1}$. Then we choose $X^{\prime}=\operatorname{Tr} D A_{j}$ and $Z=A_{j-1} \oplus \operatorname{Tr} D B_{3}$. In the second case, we have

$$
\left[U, B_{3}\right]-\left[M, B_{3}\right]=0
$$

For $M_{1}=0$ one gets $\left[M_{2}, B_{3}\right] \neq 0$ immediately. In the other case one obtains

$$
\left[U, B_{3}\right]-\left[M_{1}, B_{3}\right]=\left[R, B_{3}\right]^{1}-\left[M_{1}, B_{3}\right]^{1}=\left[\operatorname{Tr} D B_{3}, R\right] \neq 0,
$$

whence again $\left[M_{2}, B_{3}\right] \neq 0$. Thus there is a smallest index $j \geq 1$ such that $B_{j}$ is a direct summand of $M_{2}$. Then $X^{\prime}=B_{j}$ and $Z=A_{3} \oplus \operatorname{Tr} D B_{j-1}$ solve our problem. Here we set $B_{0}=A_{0}$.

For $\tilde{E}_{8}$, three cases remain to be done. First consider $X=C_{1}$. Then we have $\varepsilon\left(A_{0}\right) \geq 6$, whence $\varepsilon$ vanishes at most in $A_{5}$. The arguments used in the last case can be applied again. Furthermore, the case $X=B_{1}$ is quite similar to the case $X=A_{j}$. 
So we finally analyze in detail the most complicated case $X=B_{2}$. We want to replace $X$ by $A_{5} \oplus \operatorname{Tr} D^{7} A_{5}$, as shown in the figure below.

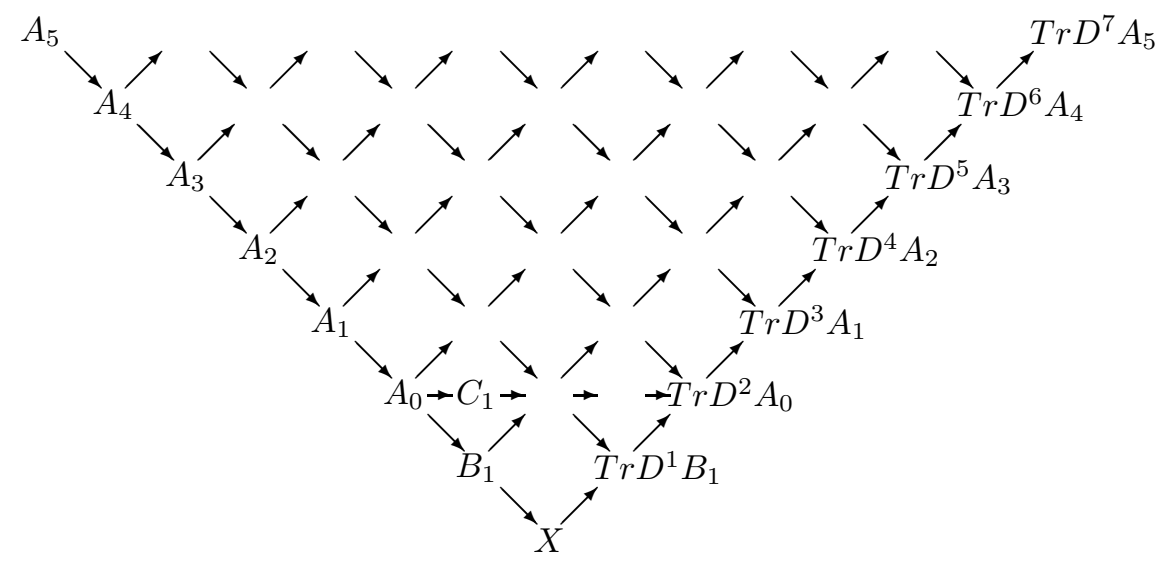

This is possible provided $\varepsilon$ does not vanish on $S$ or on $\operatorname{Tr} D^{i+1} A_{i}$ for some $i=0,1, \ldots, 5$. Because $\varepsilon\left(B_{1}\right) \geq 4$ and because of the root test, $\varepsilon$ vanishes at most in $A_{5}$ and in $\operatorname{Tr} D^{6} A_{5}$. If $\operatorname{Tr} D A_{0}$ is a direct summand of $M$, we put $X^{\prime}=\operatorname{Tr} D A_{0}$ and $Z=B_{1} \oplus \operatorname{Tr} D B_{1}$. So we assume from now on that $\operatorname{Tr} D A_{0}$ is not a summand of $M$.

If $\varepsilon$ vanishes in $A_{5}$ but not in $\operatorname{Tr} D^{6} A_{5}$, one gets $\left[\operatorname{Tr} D A_{5}, M_{1}\right] \neq 0$, and one can take the smallest index $j$ such that $\operatorname{Tr} D A_{j}$ is a direct summand of $M_{1}$. The case $j=0$ has just been done, and for $j>0$ we take $X^{\prime}=\operatorname{Tr} D A_{j}$ and $Z=$ $A_{j-1} \oplus \operatorname{Tr} D^{7} A_{5}$.

If $\varepsilon$ vanishes in $\operatorname{Tr} D^{6} A_{5}$ but not in $A_{5}$, one has $\left[M_{2}, \operatorname{Tr} D^{6} A_{5}\right] \neq 0$, because otherwise one gets $0=\left[U, \operatorname{Tr} D^{6} A_{5}\right]-\left[M_{1}, \operatorname{Tr} D^{6} A_{5}\right]=\left[R, \operatorname{Tr} D^{6} A_{5}\right]^{1}$. So one of the modules $C_{1} \prec \operatorname{Tr} D^{2} A_{1} \prec \operatorname{Tr} D^{3} A_{2} \prec \operatorname{Tr} D^{4} A_{3} \prec \operatorname{Tr} D^{5} A_{4} \prec \operatorname{Tr} D^{6} A_{5}$ is a direct summand of $M_{2}$, and we choose for $W$ the $\preceq$-smallest of these modules. We always take $X^{\prime}=W$. For $X^{\prime}=C_{1}$ we set $Z=A_{5} \oplus \operatorname{Tr} D B_{2}$. If $X^{\prime}$ equals $\operatorname{Tr} D^{i+1} A_{i}$, one defines $Z$ as $Z=A_{5} \oplus \operatorname{Tr} D^{i+1} A_{i-1}$.

Finally, it is also possible that we have a direct summand $\operatorname{Tr} D A_{j}$ of $M_{1}$ as well as a direct summand $W$ of $M_{2}$. The case where $\operatorname{Tr} D A_{0}$ is a direct summand of $M$ is already solved. In all the other cases one defines $X^{\prime}=\operatorname{Tr} D A_{j} \oplus W$. For $W=C_{1}$ one has $Z=A_{j-1} \oplus \operatorname{Tr} D B_{2}$, and for $W=\operatorname{Tr} D^{i+1} A_{i}$ one sets $Z=A_{j-1} \oplus \operatorname{Tr} D^{i+1} A_{i-1}$.

For quivers of type $\tilde{A}_{n}$ or $\tilde{D}_{n}$ the proof is similar, but much easier. Namely, for $\tilde{A}_{n}$ all indecomposable preprojectives have defect -1 , so that the lemma is trivial. For $\tilde{D}_{n}$ we argue as in the previous cases, using the following notation.

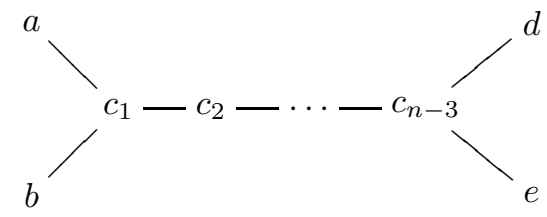

Let $X$ be a $\preceq$-minimal direct summand of $M_{2}$ with $d(U, X) \geq 2(p+d)$ having defect -2 . Then $X$ belongs to the $\operatorname{Tr} D$-orbit of some $P\left(c_{i}\right)$, and we choose $X$ so that the index $i$ is as small as possible. Using the same notational conventions as 
before, we consider the slice through $X$ with $A$ as the only source. If the "triangle" $T$ in the Auslander-Reiten quiver consisting of all $Y$ with $A \preceq Y \preceq \operatorname{Tr} D^{i-2} B$ and of $Y=\operatorname{Tr} D^{i-1} A$ contains no direct summand of $M_{2}$, one gets $\varepsilon(\bar{Y}) \geq 1$ for all $Y$ in $T$. Thus one has $M=M^{\prime} \oplus X<M^{\prime} \oplus A \oplus Z<U \oplus V$, where $Z=\operatorname{Tr} D^{i-1} B$ or $Z=\operatorname{Tr} D^{i} A$, depending on the parity of $i$.

If $T$ contains a direct summand $Y$ of $M_{2}$, then $Y$ has defect -1 by the minimal choice of $i$. Then we choose such a $Y$ that is $\preceq$-minimal, and we obtain $M=$ $M^{\prime} \oplus X \oplus Y<M^{\prime} \oplus Z \oplus W<U \oplus V$. Here $Z=C_{j}$ is the unique $\preceq$-maximal predecessor of $X$ and $Y$, and $W$ lies in the $\operatorname{Tr} D$-orbit of $A$ or $B$, depending on the parities.

\subsection{The periodicity.}

Theorem 2. Let $U$ and $V$ be two indecomposable preprojective representations over a tame quiver satisfying $d(U, V) \geq 4(p+d)$. Then the following are true:

a) Any minimal degeneration $M<U \oplus V$ admits a unique directed decomposition $M=M_{1} \oplus M_{2}$ with $d(U, X)<2(p+d)$ for all direct summands of $M_{1}$ and with $d(X, V)<2(p+d)$ for all direct summands of $M_{2}$. Here one has $\partial M_{1}=\partial U$ and $\partial M_{2}=\partial V$.

b) The map $M=M_{1} \oplus M_{2} \mapsto M_{1} \oplus \operatorname{Tr} D^{p} M_{2}$ is a bijection between the minimal degenerations $M$ to $U \oplus V$ and those to $U \oplus \operatorname{Tr} D^{p} V$. This bijection respects the codimensions.

Proof. a) Let $M_{1}$ be the direct sum of all indecomposable summands $W$ of $M$, counted with multiplicities, such that $d(U, W)<2(p+d)$. Suppose we have $\partial M_{1}>$ $\partial U$. By lemma 4, all indecomposable summands $X$ of $M$ with $d(U, X) \geq 2(p+d)$ satisfy $\partial X=-1$. By adding successively enough of these to $M_{1}$ we obtain a directed decomposition $M=M_{1}^{\prime} \oplus M_{2}^{\prime}$ with $\partial M_{1}^{\prime}=\partial U$. Then lemma 3 says

$$
\underline{\operatorname{dim}} M_{1}^{\prime}-\underline{\operatorname{dim}} U \leq \underline{n} \text {. }
$$

But $M_{1}^{\prime}$ contains at least one indecomposable $X$ with $d(U, X) \geq 2(p+d)$, which implies

Now the contradiction

$$
\underline{\operatorname{dim}} X=\underline{n}+\underline{\operatorname{dim}} D \operatorname{Tr}^{p} X .
$$

$$
\underline{\operatorname{dim}} D \operatorname{Tr}^{p} X \leq \underline{\operatorname{dim}} U
$$

implies $\partial M_{1} \leq \partial U$.

Dually, if we define $M_{2}$ as the direct sum of all summands $W$ of $M$ with $d(W, V)<2(p+d)$, we have $\partial M_{2} \leq \partial V$. By construction, and because $d(U, V) \geq$ $4(p+d), M_{1}$ and $M_{2}$ have no common direct summand. Hence $M_{1} \oplus M_{2}$ is a direct summand of $M$. We obtain

$$
\partial M=\partial(U \oplus V) \geq \partial\left(M_{1} \oplus M_{2}\right) \geq \partial M,
$$

whence $M=M_{1} \oplus M_{2}$, as desired. The uniqueness, up to isomorphism, of this decomposition is obvious.

b) Let

$$
0 \rightarrow U \rightarrow M_{1} \rightarrow R \rightarrow 0
$$

be the exact sequences as in theorem 1 with regular indecomposable end $R$. Then we get the following formulas for $\varepsilon(T)=[U \oplus V, T]-[M, T]$, where $T$ is an indecomposable. As long as $d(U, T)<2(p+d)$ holds, we have $\varepsilon(T)=[U, T]-\left[M_{1}, T\right]$. By applying $\operatorname{Hom}(, T)$ to the above exact sequence we get $\varepsilon(T)=[U, T]-\left[M_{1}, T\right]=[R, T]^{1}$ 
for all $T$ with $2(p+d) \leq d(U, T) \leq d(U, V)-2(p+d)$. In all the remaining cases we have $\varepsilon(T)=[U \oplus V, T]-[M, T]=[\operatorname{Tr} D T, U \oplus V]-[\operatorname{Tr} D T, M]=$ $[\operatorname{Tr} D T, V]-\left[\operatorname{Tr} D T, M_{2}\right]$.

Let us compare $\varepsilon$ with the function $\tilde{\varepsilon}(T)=\left[U \oplus \operatorname{Tr} D^{p} V, T\right]-\left[M_{1} \oplus \operatorname{Tr} D^{p} M_{2}, T\right]$. First of all, the equality

$$
\underline{\operatorname{dim}} V=\underline{\operatorname{dim}} M_{2}+\underline{\operatorname{dim}} R
$$

implies (because $\underline{\operatorname{dim}} \operatorname{Tr} D^{p} R=\underline{\operatorname{dim}} R$ )

$$
\underline{\operatorname{dim}} \operatorname{Tr} D^{p} V=\underline{\operatorname{dim}} \operatorname{Tr} D^{p} M_{2}+\underline{\operatorname{dim}} R
$$

and therefore also $\underline{\operatorname{dim}}\left(U \oplus \operatorname{Tr} D^{p} V\right)=\underline{\operatorname{dim}}\left(M_{1} \oplus \operatorname{Tr} D^{p} M_{2}\right)$. For $d(U, T)<2(p+d)$ we have $\tilde{\varepsilon}(T)=\varepsilon(T)$. If $2(p+d) \leq d(U, T) \leq d\left(U, \operatorname{Tr} D^{p} V\right)-2(p+d)$, one obtains $\tilde{\varepsilon}(T)=[R, T]^{1} \geq 0$. Finally, in all the remaining cases for $T=\operatorname{Tr} D^{p} T^{\prime}$, one has $\tilde{\varepsilon}(T)=\left[\operatorname{Tr} D T, \operatorname{Tr} D^{p} V\right]-\left[\operatorname{Tr} D T, \operatorname{Tr} D^{p} M_{2}\right]=\varepsilon\left(T^{\prime}\right)$. Thus we have shown at least that $M_{1} \oplus \operatorname{Tr} D^{p} M_{2}$ degenerates to $U \oplus \operatorname{Tr} D^{p} V$.

If this is not a minimal degeneration, there is one $M_{1}^{\prime} \oplus M_{2}^{\prime}$ in between, where by part a) the direct summands of $M_{1}^{\prime}$, resp. $M_{2}^{\prime}$, have distance at most $2(p+d)$ from $U$, resp. $\operatorname{Tr} D^{p} V$. One sees by similar trivial calculations as above that $M_{1}^{\prime} \oplus D \operatorname{Tr}^{p} M_{2}^{\prime}$ is then a degeneration properly between the original $M$ and $U \oplus V$, a contradiction. The same argument shows that the image of the injective map $M=M_{1} \oplus M_{2} \mapsto$ $M_{1} \oplus \operatorname{Tr} D^{p} M_{2}$ is the set of all minimal degenerations to $U \oplus \operatorname{Tr} D^{p} V$.

Finally, the codimensions are preserved, because we have

$$
\begin{aligned}
& {[U \oplus V, U \oplus V]-[M, M] } \\
&=[U \oplus V, M]-[U \oplus V, M]+[U \oplus V, M]-[M, M] \\
&= 1+\left[U \oplus V, M_{1}\right]-\left[M, M_{1}\right]+\left[U \oplus V, M_{2}\right]-\left[M, M_{2}\right] \\
&= 1+\left[U, M_{1}\right]-\left[M_{1}, M_{1}\right]+\left[\operatorname{Tr} D M_{2}, V\right]-\left[\operatorname{Tr} D M_{2}, M_{2}\right] \\
&= 1+\left[U, M_{1}\right]-\left[M_{1}, M_{1}\right]+\left[\operatorname{Tr} D \operatorname{Tr} D^{p} M_{2}, \operatorname{Tr} D^{p} V\right] \\
&-\left[\operatorname{Tr} D \operatorname{Tr} D^{p} M_{2}, \operatorname{Tr} D^{p} M_{2}\right]=\cdots \\
&= {\left[U \oplus \operatorname{Tr} D^{p} V, U \oplus \operatorname{Tr} D^{p} V\right]-\left[M_{1} \oplus \operatorname{Tr} D^{p} M_{2}, M_{1} \oplus \operatorname{Tr} D^{p} M_{2}\right] . }
\end{aligned}
$$

\section{The NUMERICAL RESUlts}

4.1. Some remarks on the program. Given two indecomposables $U \prec V$ in a preprojective component of some path algebra $k Q$, one has the following obvious algorithm to determine the set of predecessors of $U \oplus V$ with respect to the degeneration order.

- S1: Determine the finite set $K_{1}$ of all modules $M$ with $\underline{\operatorname{dim}} M=\underline{\operatorname{dim} U} \oplus V$ such that all indecomposable direct summands $X$ of $M$ satisfy $U \prec X \prec V$.

- S2: Consider then the subset $K_{2}$ of $K_{1}$ that contains only the modules $M$ satisfying $[M, X] \leq[U \oplus V, X]$ for all indecomposables $X$ with $U \prec X \prec V$. These are just all modules $M \neq U \oplus V$ degenerating to $U \oplus V$.

- S3: Determine the wanted set $K_{3}$ of all immediate predecessors $M<U \oplus V$.

The trouble with this naive algorithm is that the small set $K_{3}$ we are interested in is constructed with the help of the much bigger sets $K_{1}$ and $K_{2}$. So we refined the algorithm as follows:

- R1: The candidates for $M$ are built by adding a new indecomposable direct summand $X$ to a "partial candidate" $\tilde{M}$, and then one checks immediately 
whether $[\tilde{M} \oplus X, Y] \leq[U \oplus V, Y]$ holds for all $Y$ or not. Thus one constructs only $K_{2}$.

- R2: For each element $M$ of $K_{2}$ one considers the codimension

$$
c(M)=[U \oplus V, U \oplus V]-[M, M] .
$$

Then modules with codimension one are automatically minimal extensions, and it is much easier to find afterwards all the remaining minimal extensions.

But even this refined algorithm is not nearly fast enough to perform the calculations necessary for type $\tilde{E}_{8}$ in a reasonable time on a personal computer. Apart from some additional tricks described in 8, the root test of section 2.2 makes the algorithm work for tame quivers. This is by chance, because theorem 1 gives only a necessary condition for minimal degenerations. However, the root test can be checked immediately after the addition of a new direct summand as in R1, and it turns out that most of the nonminimal degenerations are thrown away immediately.

Of course, there might be mistakes in our program, but the following facts indicate that it is correct:

a) We confirmed the results found by U. Markolf in [10] for Dynkin quivers (within a few seconds).

b) We did not implement symmetries of the quiver into our program, but the results reflected all symmetries and dualities.

c) We checked some small cases by hand.

d) We checked the periodicity guaranteed by theorem 2, not only by comparing the numbers, but also by verifying the bijection described in theorem 2 .

4.2. Some facts found by computer, and some tables. The best of these results is that the codimensions of the minimal extensions are at most 2. By theoretical means we could only show that $1-\partial(U \oplus V)$ is an upper bound.

Next, the numbers $e(U, V)$, resp. $c(U, V)$, of all minimal extensions between $U$, resp. $V$, of those with codimension 2 are bounded by 613 , resp. 76 , and both bounds are reached for certain representations of an $\tilde{E}_{8}$-quiver. Moreover, the highest multiplicity of a direct summand occurring in $M$ for some minimal degeneration $M<U \oplus V$ is 2 , and there are at most 12 nonisomorphic indecomposable direct summands. The last statement is an easy consequence of the additivity of the defect in short exact sequences.

At the end of this article we include some tables to indicate the combinatorial complexity of the problem we are dealing with. For more details we refer to [8] as well as to http://wmaz.math.uni-wuppertal.de.

First of all, we give no table for type $\tilde{A}_{n}$, because there are always at most two possible minimal extensions, both having two indecomposable direct summands. If one cannot reduce to the double-arrow by shrinking some arrows, the codimension is one. So this case is trivial.

The tables included for type $\tilde{D}_{8}$ contain the following information. In each table, we fix the simple projective $P(\omega)$ and we look at the slice $S$ in the AuslanderReiten quiver with $\omega$ as the only source. Of course, up to symmetry and duality, we only have to choose $a, c_{1}, c_{2}$ and $c_{3}$ for $\omega$. Furthermore, for $\omega=c_{1}$ the points $a, b$ and the points $c, d$ are symmetric, so that we have to consider only one of these. Similar remarks apply to the other choices of $\omega$. The slice $S$ contains the indecomposable projectives $P(x)$ to all the points in $Q$. The first row of the table shows the labels $a, b, c_{1}, c_{2}, \ldots, c_{5}, d, e$ of these points as in section 3.3. The two 
entries standing in the $i+1^{\text {th }}$ row under the label $x$ are the numbers $e\left(U, \operatorname{Tr} D^{i} P(x)\right)$ and $c\left(U, \operatorname{Tr} D^{i} P(x)\right)$ separated by a slash. Now in each table, two rows are separated by a small gap. At this step the periodic behaviour guaranteed by theorem 2 starts. The length of the period coincides always with the Coxeter number $p=6$ of $\tilde{D}_{8}$, but the periodic behaviour starts at different levels depending on $\omega$. The smallest index that works for all $\omega$ 's is $13=2 p+1$. Thus theorem 2 is best possible for the length of the period, but not for the starting point. Each table stops 6 steps after the periodic behaviour has started.

The tables for the other types are similar. Again we have used the symmetries. To save even more space, we have skipped the somewhat nonregular beginnings and we have given the numbers only from the smallest index where one has the periodic behaviour. This index is always $2 p+1$, where $p=6$, resp. $p=12$, resp. $p=30$, is the Coxeter-number of type $\tilde{E}_{6}$, resp. $\tilde{E}_{7}$, resp. $\tilde{E}_{8}$.

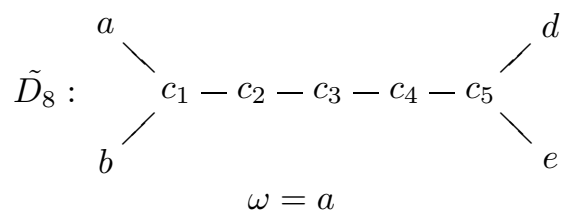

\begin{tabular}{|c||c|c|c|c|c|c|c|c|}
\hline & $a$ & $b$ & $c_{1}$ & $c_{2}$ & $c_{3}$ & $c_{4}$ & $c_{5}$ & $d$ \\
\hline \hline 1 & $1 / 0$ & $1 / 0$ & $1 / 0$ & $1 / 0$ & $1 / 0$ & $1 / 0$ & $1 / 0$ & $1 / 0$ \\
\hline 2 & $0 / 0$ & $0 / 0$ & $1 / 0$ & $1 / 0$ & $1 / 0$ & $1 / 0$ & $3 / 0$ & $1 / 0$ \\
\hline 3 & $1 / 0$ & $1 / 0$ & $1 / 0$ & $1 / 0$ & $1 / 0$ & $3 / 0$ & $3 / 0$ & $1 / 0$ \\
\hline 4 & $0 / 0$ & $0 / 0$ & $1 / 0$ & $1 / 0$ & $3 / 0$ & $3 / 0$ & $3 / 0$ & $1 / 0$ \\
\hline 5 & $1 / 0$ & $1 / 0$ & $1 / 0$ & $3 / 0$ & $3 / 0$ & $3 / 0$ & $3 / 0$ & $1 / 0$ \\
\hline 6 & $0 / 0$ & $1 / 0$ & $3 / 0$ & $3 / 0$ & $3 / 0$ & $3 / 0$ & $3 / 0$ & $1 / 0$ \\
\hline 7 & $3 / 0$ & $3 / 0$ & $5 / 0$ & $5 / 0$ & $5 / 0$ & $5 / 0$ & $4 / 0$ & $2 / 0$ \\
\hline 8 & $1 / 0$ & $1 / 0$ & $5 / 0$ & $5 / 0$ & $5 / 0$ & $4 / 0$ & $5 / 0$ & $2 / 0$ \\
\hline 9 & $3 / 0$ & $3 / 0$ & $5 / 0$ & $5 / 0$ & $4 / 0$ & $5 / 0$ & $5 / 0$ & $2 / 0$ \\
\hline 10 & $1 / 0$ & $1 / 0$ & $5 / 0$ & $4 / 0$ & $5 / 0$ & $5 / 0$ & $5 / 0$ & $2 / 0$ \\
\hline 11 & $3 / 0$ & $2 / 0$ & $4 / 0$ & $5 / 0$ & $5 / 0$ & $5 / 0$ & $5 / 0$ & $2 / 0$ \\
\hline 12 & $1 / 0$ & $1 / 0$ & $3 / 0$ & $3 / 0$ & $3 / 0$ & $3 / 0$ & $3 / 0$ & $1 / 0$ \\
\hline \hline 13 & $3 / 0$ & $3 / 0$ & $5 / 0$ & $5 / 0$ & $5 / 0$ & $5 / 0$ & $4 / 1$ & $2 / 0$ \\
\hline 14 & $1 / 0$ & $1 / 0$ & $5 / 0$ & $5 / 0$ & $5 / 0$ & $4 / 1$ & $5 / 0$ & $2 / 0$ \\
\hline 15 & $3 / 0$ & $3 / 0$ & $5 / 0$ & $5 / 0$ & $4 / 1$ & $5 / 0$ & $5 / 0$ & $2 / 0$ \\
\hline 16 & $1 / 0$ & $1 / 0$ & $5 / 0$ & $4 / 1$ & $5 / 0$ & $5 / 0$ & $5 / 0$ & $2 / 0$ \\
\hline 17 & $3 / 0$ & $2 / 0$ & $4 / 1$ & $5 / 0$ & $5 / 0$ & $5 / 0$ & $5 / 0$ & $2 / 0$ \\
\hline 18 & $1 / 1$ & $1 / 0$ & $3 / 0$ & $3 / 0$ & $3 / 0$ & $3 / 0$ & $3 / 0$ & $1 / 0$ \\
\hline
\end{tabular}




$$
\omega=c_{1}
$$

\begin{tabular}{|c||c|c|c|c|c|c|c|}
\hline & $a$ & $c_{1}$ & $c_{2}$ & $c_{3}$ & $c_{4}$ & $c_{5}$ & $d$ \\
\hline \hline 1 & $1 / 0$ & $1 / 0$ & $1 / 0$ & $1 / 0$ & $1 / 0$ & $1 / 0$ & $1 / 0$ \\
\hline 2 & $1 / 0$ & $3 / 0$ & $3 / 0$ & $3 / 0$ & $3 / 0$ & $6 / 0$ & $3 / 0$ \\
\hline 3 & $1 / 0$ & $3 / 0$ & $3 / 0$ & $3 / 0$ & $6 / 0$ & $9 / 0$ & $3 / 0$ \\
\hline 4 & $1 / 0$ & $3 / 0$ & $3 / 0$ & $6 / 0$ & $9 / 0$ & $9 / 0$ & $3 / 0$ \\
\hline 5 & $1 / 0$ & $3 / 0$ & $6 / 0$ & $9 / 0$ & $9 / 0$ & $9 / 0$ & $3 / 0$ \\
\hline 6 & $3 / 0$ & $6 / 0$ & $9 / 0$ & $9 / 0$ & $9 / 0$ & $9 / 0$ & $3 / 0$ \\
\hline 7 & $5 / 0$ & $12 / 0$ & $12 / 0$ & $12 / 0$ & $12 / 0$ & $10 / 0$ & $4 / 0$ \\
\hline 8 & $5 / 0$ & $13 / 0$ & $13 / 0$ & $13 / 0$ & $11 / 0$ & $10 / 0$ & $5 / 0$ \\
\hline 9 & $5 / 0$ & $13 / 0$ & $13 / 0$ & $11 / 0$ & $10 / 0$ & $13 / 0$ & $5 / 0$ \\
\hline 10 & $5 / 0$ & $13 / 0$ & $11 / 0$ & $10 / 0$ & $13 / 0$ & $13 / 0$ & $5 / 0$ \\
\hline 11 & $4 / 0$ & $11 / 0$ & $10 / 0$ & $13 / 0$ & $13 / 0$ & $13 / 0$ & $5 / 0$ \\
\hline \hline 12 & $3 / 0$ & $6 / 0$ & $9 / 0$ & $9 / 0$ & $9 / 0$ & $9 / 0$ & $3 / 0$ \\
\hline 13 & $5 / 0$ & $12 / 2$ & $12 / 2$ & $12 / 2$ & $12 / 2$ & $10 / 4$ & $4 / 1$ \\
\hline 14 & $5 / 0$ & $13 / 0$ & $13 / 0$ & $13 / 0$ & $11 / 2$ & $10 / 0$ & $5 / 0$ \\
\hline 15 & $5 / 0$ & $13 / 0$ & $13 / 0$ & $11 / 2$ & $10 / 0$ & $13 / 0$ & $5 / 0$ \\
\hline 16 & $5 / 0$ & $13 / 0$ & $11 / 2$ & $10 / 0$ & $13 / 0$ & $13 / 0$ & $5 / 0$ \\
\hline 17 & $4 / 1$ & $11 / 2$ & $10 / 0$ & $13 / 0$ & $13 / 0$ & $13 / 0$ & $5 / 0$ \\
\hline
\end{tabular}

$$
\omega=c_{2}
$$

\begin{tabular}{|c||c|c|c|c|c|c|c|}
\hline & $a$ & $c_{1}$ & $c_{2}$ & $c_{3}$ & $c_{4}$ & $c_{5}$ & $d$ \\
\hline \hline 1 & $1 / 0$ & $1 / 0$ & $1 / 0$ & $1 / 0$ & $1 / 0$ & $1 / 0$ & $1 / 0$ \\
\hline 2 & $1 / 0$ & $3 / 0$ & $1 / 0$ & $1 / 0$ & $1 / 0$ & $3 / 0$ & $1 / 0$ \\
\hline 3 & $1 / 0$ & $3 / 0$ & $3 / 0$ & $3 / 0$ & $6 / 0$ & $6 / 0$ & $3 / 0$ \\
\hline 4 & $1 / 0$ & $3 / 0$ & $3 / 0$ & $6 / 0$ & $6 / 0$ & $9 / 0$ & $3 / 0$ \\
\hline 5 & $3 / 0$ & $6 / 0$ & $6 / 0$ & $6 / 0$ & $9 / 0$ & $9 / 0$ & $3 / 0$ \\
\hline 6 & $3 / 0$ & $9 / 0$ & $6 / 0$ & $9 / 0$ & $9 / 0$ & $9 / 0$ & $3 / 0$ \\
\hline 7 & $5 / 0$ & $12 / 0$ & $14 / 0$ & $14 / 0$ & $14 / 0$ & $12 / 0$ & $5 / 0$ \\
\hline 8 & $5 / 0$ & $13 / 0$ & $12 / 0$ & $12 / 0$ & $10 / 0$ & $11 / 0$ & $4 / 0$ \\
\hline 9 & $5 / 0$ & $13 / 0$ & $13 / 0$ & $11 / 0$ & $14 / 0$ & $10 / 0$ & $5 / 0$ \\
\hline 10 & $4 / 0$ & $11 / 0$ & $11 / 0$ & $14 / 0$ & $10 / 0$ & $13 / 0$ & $5 / 0$ \\
\hline \hline 11 & $5 / 0$ & $10 / 0$ & $14 / 0$ & $10 / 0$ & $13 / 0$ & $13 / 0$ & $5 / 0$ \\
\hline 12 & $3 / 0$ & $9 / 0$ & $6 / 0$ & $9 / 0$ & $9 / 0$ & $9 / 0$ & $3 / 0$ \\
\hline 13 & $5 / 0$ & $12 / 2$ & $14 / 0$ & $14 / 0$ & $14 / 0$ & $12 / 2$ & $5 / 0$ \\
\hline 14 & $5 / 0$ & $13 / 0$ & $12 / 2$ & $12 / 2$ & $10 / 4$ & $11 / 2$ & $4 / 1$ \\
\hline 15 & $5 / 0$ & $13 / 0$ & $13 / 0$ & $11 / 2$ & $14 / 0$ & $10 / 0$ & $5 / 0$ \\
\hline 16 & $4 / 1$ & $11 / 2$ & $11 / 2$ & $14 / 0$ & $10 / 0$ & $13 / 0$ & $5 / 0$ \\
\hline
\end{tabular}




$$
\omega=c_{3}
$$

\begin{tabular}{|c||c|c|c|c|c|c|c|}
\hline & $a$ & $c_{1}$ & $c_{2}$ & $c_{3}$ & $c_{4}$ & $c_{5}$ & $d$ \\
\hline \hline 1 & $1 / 0$ & $1 / 0$ & $1 / 0$ & $1 / 0$ & $1 / 0$ & $1 / 0$ & $1 / 0$ \\
\hline 2 & $1 / 0$ & $3 / 0$ & $1 / 0$ & $1 / 0$ & $1 / 0$ & $3 / 0$ & $1 / 0$ \\
\hline 3 & $1 / 0$ & $3 / 0$ & $3 / 0$ & $1 / 0$ & $3 / 0$ & $3 / 0$ & $1 / 0$ \\
\hline 4 & $3 / 0$ & $6 / 0$ & $6 / 0$ & $6 / 0$ & $6 / 0$ & $6 / 0$ & $3 / 0$ \\
\hline 5 & $3 / 0$ & $9 / 0$ & $6 / 0$ & $6 / 0$ & $6 / 0$ & $9 / 0$ & $3 / 0$ \\
\hline 6 & $3 / 0$ & $9 / 0$ & $9 / 0$ & $6 / 0$ & $9 / 0$ & $9 / 0$ & $3 / 0$ \\
\hline 7 & $5 / 0$ & $12 / 0$ & $14 / 0$ & $14 / 0$ & $14 / 0$ & $12 / 0$ & $5 / 0$ \\
\hline 8 & $5 / 0$ & $13 / 0$ & $12 / 0$ & $14 / 0$ & $12 / 0$ & $13 / 0$ & $5 / 0$ \\
\hline 9 & $4 / 0$ & $11 / 0$ & $11 / 0$ & $10 / 0$ & $11 / 0$ & $11 / 0$ & $4 / 0$ \\
\hline \hline 10 & $5 / 0$ & $10 / 0$ & $14 / 0$ & $14 / 0$ & $14 / 0$ & $10 / 0$ & $5 / 0$ \\
\hline 11 & $5 / 0$ & $13 / 0$ & $10 / 0$ & $14 / 0$ & $10 / 0$ & $13 / 0$ & $5 / 0$ \\
\hline 12 & $3 / 0$ & $9 / 0$ & $9 / 0$ & $6 / 0$ & $9 / 0$ & $9 / 0$ & $3 / 0$ \\
\hline 13 & $5 / 0$ & $12 / 2$ & $14 / 0$ & $14 / 0$ & $14 / 0$ & $12 / 2$ & $5 / 0$ \\
\hline 14 & $5 / 0$ & $13 / 0$ & $12 / 2$ & $14 / 0$ & $12 / 2$ & $13 / 0$ & $5 / 0$ \\
\hline 15 & $4 / 1$ & $11 / 2$ & $11 / 2$ & $10 / 4$ & $11 / 2$ & $11 / 2$ & $4 / 1$ \\
\hline
\end{tabular}

$$
\begin{array}{cc} 
& a_{2}-a_{1}-a_{0}-b_{1}-b_{2} \\
\tilde{E}_{6}: & \mid \\
& c_{1} \\
& l \\
c_{2}
\end{array}
$$

$$
\omega=a_{2}
$$

\begin{tabular}{|c||c|c|c|c|c|}
\hline & $a_{2}$ & $a_{1}$ & $a_{0}$ & $b_{1}$ & $b_{2}$ \\
\hline \hline 13 & $3 / 0$ & $7 / 0$ & $9 / 0$ & $5 / 0$ & $3 / 0$ \\
\hline 14 & $2 / 0$ & $3 / 0$ & $8 / 1$ & $5 / 0$ & $1 / 0$ \\
\hline 15 & $1 / 0$ & $4 / 1$ & $10 / 1$ & $4 / 1$ & $2 / 0$ \\
\hline 16 & $2 / 0$ & $7 / 0$ & $8 / 1$ & $5 / 0$ & $2 / 0$ \\
\hline 17 & $3 / 0$ & $4 / 1$ & $9 / 0$ & $5 / 0$ & $2 / 0$ \\
\hline 18 & $1 / 1$ & $3 / 0$ & $7 / 0$ & $3 / 0$ & $1 / 0$ \\
\hline
\end{tabular}

$\omega=a_{1}$

\begin{tabular}{|c||c|c|c|c|c|}
\hline & $a_{2}$ & $a_{1}$ & $a_{0}$ & $b_{1}$ & $b_{2}$ \\
\hline \hline 13 & $7 / 0$ & $15 / 1$ & $23 / 3$ & $12 / 2$ & $5 / 0$ \\
\hline 14 & $3 / 0$ & $12 / 0$ & $24 / 4$ & $14 / 1$ & $5 / 0$ \\
\hline 15 & $4 / 1$ & $10 / 4$ & $23 / 3$ & $11 / 2$ & $4 / 1$ \\
\hline 16 & $7 / 0$ & $14 / 0$ & $21 / 1$ & $10 / 0$ & $5 / 0$ \\
\hline 17 & $4 / 1$ & $13 / 1$ & $20 / 0$ & $13 / 0$ & $5 / 0$ \\
\hline 18 & $3 / 0$ & $6 / 0$ & $21 / 1$ & $9 / 0$ & $3 / 0$ \\
\hline
\end{tabular}

$\omega=a_{0}$

\begin{tabular}{|c||c|c|c|}
\hline & $a_{2}$ & $a_{1}$ & $a_{0}$ \\
\hline \hline 13 & $9 / 0$ & $23 / 3$ & $46 / 6$ \\
\hline 14 & $8 / 1$ & $24 / 4$ & $45 / 9$ \\
\hline 15 & $10 / 1$ & $23 / 3$ & $46 / 6$ \\
\hline 16 & $8 / 1$ & $21 / 1$ & $39 / 3$ \\
\hline 17 & $9 / 0$ & $20 / 0$ & $40 / 0$ \\
\hline 18 & $7 / 0$ & $21 / 1$ & $39 / 3$ \\
\hline
\end{tabular}




$$
\tilde{E}_{7}: \begin{gathered}
a_{3}-a_{2}-a_{1}-a_{0}-b_{1}-b_{2}-b_{3} \\
c_{1} \\
\omega=c_{1}
\end{gathered}
$$

\begin{tabular}{|c||c|c|c|c|c|}
\hline & $a_{3}$ & $a_{2}$ & $a_{1}$ & $a_{0}$ & $c_{1}$ \\
\hline \hline 25 & $7 / 0$ & $15 / 1$ & $27 / 1$ & $43 / 2$ & $18 / 0$ \\
\hline 26 & $3 / 0$ & $12 / 0$ & $23 / 3$ & $39 / 4$ & $9 / 0$ \\
\hline 27 & $5 / 0$ & $12 / 2$ & $28 / 2$ & $42 / 6$ & $14 / 0$ \\
\hline 28 & $5 / 0$ & $14 / 1$ & $24 / 4$ & $45 / 4$ & $12 / 2$ \\
\hline 29 & $5 / 0$ & $14 / 1$ & $28 / 2$ & $41 / 6$ & $17 / 0$ \\
\hline 30 & $4 / 1$ & $11 / 2$ & $23 / 3$ & $40 / 4$ & $10 / 4$ \\
\hline 31 & $7 / 0$ & $14 / 0$ & $27 / 1$ & $43 / 2$ & $18 / 0$ \\
\hline 32 & $4 / 1$ & $13 / 1$ & $21 / 1$ & $37 / 2$ & $11 / 2$ \\
\hline 33 & $5 / 0$ & $10 / 0$ & $26 / 0$ & $36 / 0$ & $14 / 0$ \\
\hline 34 & $5 / 0$ & $13 / 0$ & $20 / 0$ & $41 / 0$ & $10 / 0$ \\
\hline 35 & $5 / 0$ & $13 / 0$ & $26 / 0$ & $35 / 0$ & $17 / 0$ \\
\hline 36 & $3 / 0$ & $9 / 0$ & $21 / 1$ & $38 / 2$ & $6 / 0$ \\
\hline
\end{tabular}

$$
\omega=a_{3}
$$

\begin{tabular}{|c||c|c|c|c|c|c|c|c|}
\hline & $a_{3}$ & $a_{2}$ & $a_{1}$ & $a_{0}$ & $b_{1}$ & $b_{2}$ & $b_{3}$ & $c_{1}$ \\
\hline \hline 25 & $3 / 0$ & $7 / 0$ & $12 / 0$ & $15 / 0$ & $9 / 0$ & $5 / 0$ & $3 / 0$ & $7 / 0$ \\
\hline 26 & $2 / 0$ & $5 / 0$ & $7 / 0$ & $15 / 0$ & $10 / 0$ & $7 / 0$ & $2 / 0$ & $3 / 0$ \\
\hline 27 & $2 / 0$ & $3 / 0$ & $9 / 0$ & $13 / 1$ & $9 / 0$ & $3 / 0$ & $1 / 0$ & $5 / 0$ \\
\hline 28 & $1 / 0$ & $5 / 0$ & $8 / 1$ & $16 / 1$ & $8 / 1$ & $4 / 1$ & $2 / 0$ & $5 / 0$ \\
\hline 29 & $3 / 0$ & $5 / 0$ & $12 / 0$ & $16 / 1$ & $10 / 1$ & $7 / 0$ & $3 / 0$ & $5 / 0$ \\
\hline 30 & $1 / 0$ & $5 / 0$ & $8 / 1$ & $14 / 2$ & $10 / 0$ & $5 / 0$ & $1 / 0$ & $4 / 1$ \\
\hline 31 & $3 / 0$ & $5 / 0$ & $10 / 1$ & $16 / 1$ & $10 / 1$ & $4 / 1$ & $2 / 0$ & $7 / 0$ \\
\hline 32 & $1 / 0$ & $4 / 1$ & $8 / 1$ & $16 / 1$ & $8 / 1$ & $5 / 0$ & $2 / 0$ & $4 / 1$ \\
\hline 33 & $2 / 0$ & $5 / 0$ & $12 / 0$ & $13 / 1$ & $9 / 0$ & $5 / 0$ & $2 / 0$ & $5 / 0$ \\
\hline 34 & $2 / 0$ & $7 / 0$ & $8 / 1$ & $15 / 0$ & $10 / 0$ & $5 / 0$ & $2 / 0$ & $5 / 0$ \\
\hline 35 & $3 / 0$ & $4 / 1$ & $9 / 0$ & $15 / 0$ & $9 / 0$ & $5 / 0$ & $2 / 0$ & $5 / 0$ \\
\hline 36 & $1 / 1$ & $3 / 0$ & $7 / 0$ & $12 / 0$ & $7 / 0$ & $3 / 0$ & $1 / 0$ & $3 / 0$ \\
\hline
\end{tabular}

$$
\omega=a_{2}
$$

\begin{tabular}{|c||c|c|c|c|c|c|c|c|}
\hline & $a_{3}$ & $a_{2}$ & $a_{1}$ & $a_{0}$ & $b_{1}$ & $b_{2}$ & $b_{3}$ & $c_{1}$ \\
\hline \hline 25 & $7 / 0$ & $15 / 1$ & $28 / 2$ & $39 / 4$ & $23 / 3$ & $12 / 2$ & $5 / 0$ & $15 / 1$ \\
\hline 26 & $5 / 0$ & $16 / 0$ & $24 / 0$ & $43 / 3$ & $28 / 2$ & $17 / 0$ & $7 / 0$ & $12 / 0$ \\
\hline 27 & $3 / 0$ & $9 / 0$ & $23 / 3$ & $39 / 5$ & $25 / 1$ & $12 / 0$ & $3 / 0$ & $12 / 2$ \\
\hline 28 & $5 / 0$ & $12 / 2$ & $24 / 4$ & $40 / 5$ & $24 / 4$ & $10 / 4$ & $4 / 1$ & $14 / 1$ \\
\hline 29 & $5 / 0$ & $14 / 1$ & $27 / 1$ & $43 / 3$ & $23 / 3$ & $14 / 0$ & $7 / 0$ & $14 / 1$ \\
\hline 30 & $5 / 0$ & $13 / 0$ & $26 / 2$ & $38 / 4$ & $26 / 0$ & $16 / 0$ & $5 / 0$ & $11 / 2$ \\
\hline 31 & $5 / 0$ & $14 / 1$ & $23 / 3$ & $37 / 2$ & $26 / 2$ & $11 / 2$ & $4 / 1$ & $14 / 0$ \\
\hline 32 & $4 / 1$ & $11 / 2$ & $21 / 1$ & $41 / 1$ & $21 / 1$ & $10 / 0$ & $5 / 0$ & $13 / 1$ \\
\hline 33 & $5 / 0$ & $10 / 0$ & $26 / 0$ & $35 / 1$ & $20 / 0$ & $13 / 0$ & $5 / 0$ & $10 / 0$ \\
\hline 34 & $7 / 0$ & $17 / 0$ & $25 / 1$ & $36 / 1$ & $27 / 1$ & $13 / 0$ & $5 / 0$ & $13 / 0$ \\
\hline 35 & $4 / 1$ & $13 / 1$ & $20 / 0$ & $41 / 1$ & $24 / 0$ & $13 / 0$ & $5 / 0$ & $13 / 0$ \\
\hline 36 & $3 / 0$ & $6 / 0$ & $21 / 1$ & $36 / 2$ & $21 / 1$ & $9 / 0$ & $3 / 0$ & $9 / 0$ \\
\hline
\end{tabular}




$$
\omega=a_{1}
$$

\begin{tabular}{|c||c|c|c|c|c|c|c|c|}
\hline & $a_{3}$ & $a_{2}$ & $a_{1}$ & $a_{0}$ & $b_{1}$ & $b_{2}$ & $b_{3}$ & $c_{1}$ \\
\hline \hline 25 & $12 / 0$ & $28 / 2$ & $53 / 4$ & $79 / 8$ & $46 / 6$ & $23 / 3$ & $9 / 0$ & $27 / 1$ \\
\hline 26 & $7 / 0$ & $24 / 0$ & $47 / 4$ & $81 / 10$ & $51 / 6$ & $28 / 2$ & $10 / 0$ & $23 / 3$ \\
\hline 27 & $9 / 0$ & $23 / 3$ & $51 / 4$ & $81 / 10$ & $51 / 4$ & $25 / 1$ & $9 / 0$ & $28 / 2$ \\
\hline 28 & $8 / 1$ & $24 / 4$ & $45 / 9$ & $81 / 10$ & $49 / 6$ & $24 / 4$ & $8 / 1$ & $24 / 4$ \\
\hline 29 & $12 / 0$ & $27 / 1$ & $53 / 4$ & $79 / 8$ & $46 / 6$ & $23 / 3$ & $10 / 1$ & $28 / 2$ \\
\hline 30 & $8 / 1$ & $26 / 2$ & $47 / 4$ & $77 / 6$ & $47 / 2$ & $26 / 0$ & $10 / 0$ & $23 / 3$ \\
\hline 31 & $10 / 1$ & $23 / 3$ & $51 / 4$ & $75 / 4$ & $49 / 2$ & $26 / 2$ & $10 / 1$ & $27 / 1$ \\
\hline 32 & $8 / 1$ & $21 / 1$ & $39 / 3$ & $73 / 2$ & $45 / 2$ & $21 / 1$ & $8 / 1$ & $21 / 1$ \\
\hline 33 & $12 / 0$ & $26 / 0$ & $49 / 0$ & $73 / 2$ & $40 / 0$ & $20 / 0$ & $9 / 0$ & $26 / 0$ \\
\hline 34 & $8 / 1$ & $25 / 1$ & $45 / 2$ & $73 / 2$ & $47 / 2$ & $27 / 1$ & $10 / 0$ & $20 / 0$ \\
\hline 35 & $9 / 0$ & $20 / 0$ & $47 / 0$ & $75 / 4$ & $49 / 2$ & $24 / 0$ & $9 / 0$ & $26 / 0$ \\
\hline 36 & $7 / 0$ & $21 / 1$ & $39 / 3$ & $77 / 6$ & $45 / 2$ & $21 / 1$ & $7 / 0$ & $21 / 1$ \\
\hline
\end{tabular}

$$
\omega=a_{0}
$$

\begin{tabular}{|c||c|c|c|c|c|}
\hline & $a_{3}$ & $a_{2}$ & $a_{1}$ & $a_{0}$ & $c_{1}$ \\
\hline \hline 25 & $15 / 0$ & $39 / 4$ & $79 / 8$ & $133 / 14$ & $43 / 2$ \\
\hline 26 & $15 / 0$ & $43 / 3$ & $81 / 10$ & $134 / 16$ & $39 / 4$ \\
\hline 27 & $13 / 1$ & $39 / 5$ & $81 / 10$ & $130 / 20$ & $42 / 6$ \\
\hline 28 & $16 / 1$ & $40 / 5$ & $81 / 10$ & $135 / 16$ & $45 / 4$ \\
\hline 29 & $16 / 1$ & $43 / 3$ & $79 / 8$ & $132 / 14$ & $41 / 6$ \\
\hline 30 & $14 / 2$ & $38 / 4$ & $77 / 6$ & $122 / 12$ & $40 / 4$ \\
\hline 31 & $16 / 1$ & $37 / 2$ & $75 / 4$ & $125 / 6$ & $43 / 2$ \\
\hline 32 & $16 / 1$ & $41 / 1$ & $73 / 2$ & $122 / 4$ & $37 / 2$ \\
\hline 33 & $13 / 1$ & $35 / 1$ & $73 / 2$ & $114 / 4$ & $36 / 0$ \\
\hline 34 & $15 / 0$ & $36 / 1$ & $73 / 2$ & $123 / 4$ & $41 / 0$ \\
\hline 35 & $15 / 0$ & $41 / 1$ & $75 / 4$ & $124 / 6$ & $35 / 0$ \\
\hline 36 & $12 / 0$ & $36 / 2$ & $77 / 6$ & $122 / 12$ & $38 / 2$ \\
\hline
\end{tabular}




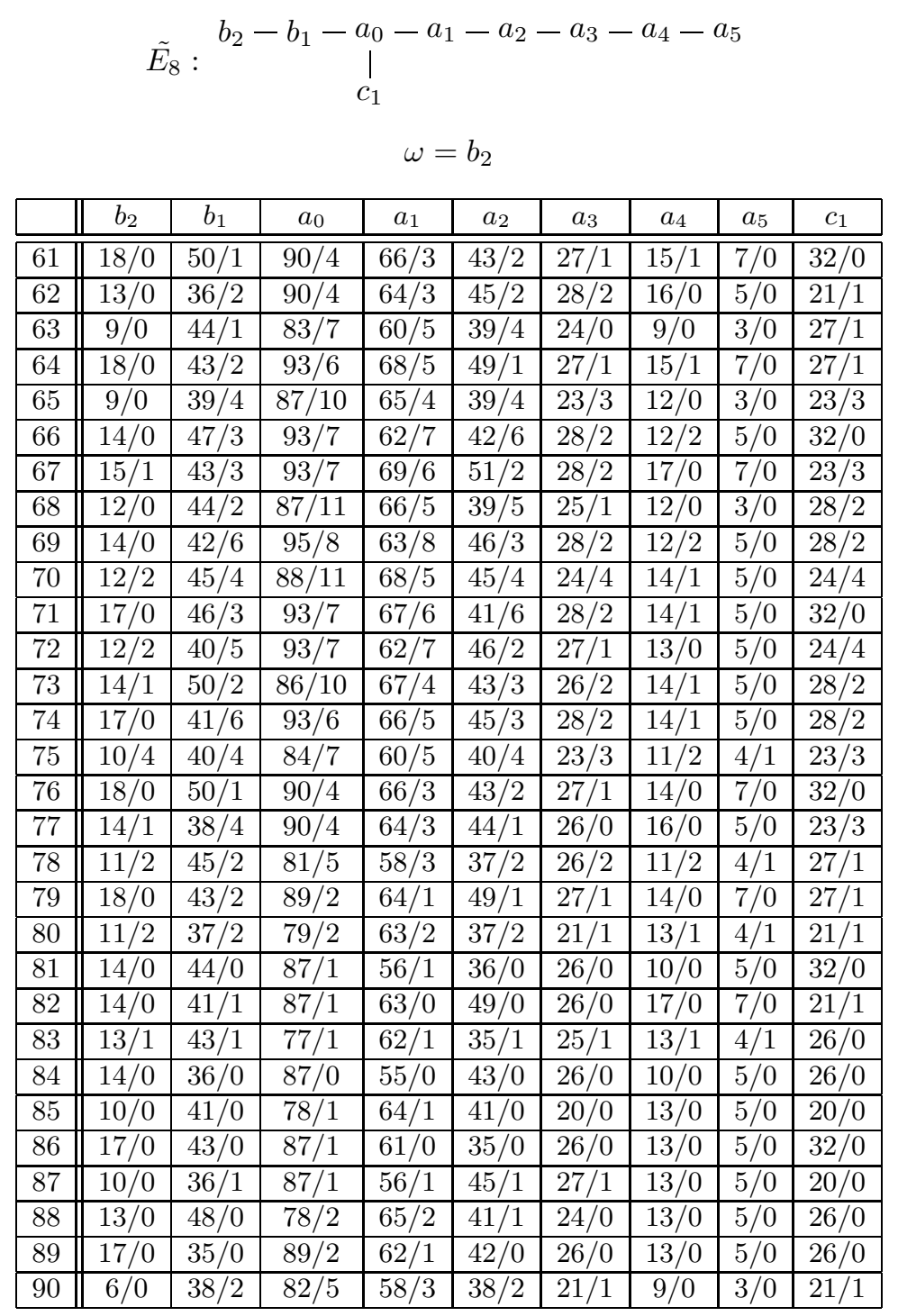




$$
\omega=b_{1}
$$

\begin{tabular}{|c||c|c|c|c|c|c|c|c|c|}
\hline & $b_{2}$ & $b_{1}$ & $a_{0}$ & $a_{1}$ & $a_{2}$ & $a_{3}$ & $a_{4}$ & $a_{5}$ & $c_{1}$ \\
\hline \hline 61 & $51 / 1$ & $145 / 10$ & $285 / 27$ & $204 / 18$ & $133 / 14$ & $79 / 8$ & $39 / 4$ & $15 / 0$ & $88 / 5$ \\
\hline 62 & $36 / 2$ & $140 / 9$ & $297 / 22$ & $214 / 18$ & $146 / 12$ & $90 / 7$ & $50 / 2$ & $19 / 0$ & $85 / 4$ \\
\hline 63 & $44 / 1$ & $132 / 10$ & $284 / 29$ & $204 / 20$ & $136 / 13$ & $83 / 4$ & $39 / 0$ & $12 / 0$ & $79 / 8$ \\
\hline 64 & $43 / 2$ & $143 / 11$ & $289 / 31$ & $208 / 22$ & $142 / 11$ & $84 / 5$ & $39 / 4$ & $15 / 0$ & $89 / 6$ \\
\hline 65 & $39 / 4$ & $134 / 16$ & $292 / 33$ & $215 / 19$ & $143 / 12$ & $81 / 10$ & $43 / 3$ & $15 / 0$ & $81 / 10$ \\
\hline 66 & $47 / 3$ & $140 / 14$ & $289 / 31$ & $206 / 22$ & $130 / 20$ & $81 / 10$ & $39 / 5$ & $13 / 1$ & $89 / 6$ \\
\hline 67 & $43 / 3$ & $144 / 12$ & $300 / 25$ & $209 / 23$ & $147 / 12$ & $89 / 6$ & $45 / 2$ & $19 / 0$ & $88 / 7$ \\
\hline 68 & $44 / 2$ & $141 / 11$ & $287 / 32$ & $214 / 18$ & $142 / 11$ & $84 / 5$ & $46 / 1$ & $15 / 0$ & $81 / 10$ \\
\hline 69 & $42 / 6$ & $139 / 16$ & $288 / 30$ & $205 / 21$ & $135 / 13$ & $86 / 7$ & $39 / 5$ & $13 / 1$ & $90 / 7$ \\
\hline 70 & $45 / 4$ & $135 / 16$ & $287 / 28$ & $205 / 19$ & $144 / 12$ & $81 / 10$ & $40 / 5$ & $16 / 1$ & $81 / 10$ \\
\hline 71 & $46 / 3$ & $144 / 10$ & $282 / 24$ & $212 / 16$ & $132 / 14$ & $79 / 8$ & $43 / 3$ & $16 / 1$ & $88 / 5$ \\
\hline 72 & $40 / 5$ & $135 / 12$ & $294 / 19$ & $201 / 17$ & $135 / 9$ & $86 / 3$ & $43 / 1$ & $16 / 0$ & $88 / 7$ \\
\hline 73 & $50 / 2$ & $141 / 10$ & $278 / 23$ & $200 / 14$ & $139 / 7$ & $84 / 5$ & $43 / 3$ & $16 / 1$ & $79 / 8$ \\
\hline 74 & $41 / 6$ & $142 / 11$ & $277 / 19$ & $207 / 11$ & $138 / 8$ & $85 / 6$ & $43 / 3$ & $16 / 1$ & $88 / 5$ \\
\hline 75 & $40 / 4$ & $122 / 12$ & $274 / 15$ & $196 / 12$ & $132 / 9$ & $77 / 6$ & $38 / 4$ & $14 / 2$ & $77 / 6$ \\
\hline 76 & $50 / 1$ & $140 / 5$ & $271 / 13$ & $196 / 10$ & $125 / 6$ & $75 / 4$ & $37 / 2$ & $16 / 1$ & $86 / 3$ \\
\hline 77 & $38 / 4$ & $137 / 6$ & $285 / 10$ & $202 / 6$ & $137 / 3$ & $84 / 1$ & $48 / 0$ & $19 / 0$ & $85 / 4$ \\
\hline 78 & $45 / 2$ & $130 / 8$ & $266 / 11$ & $192 / 8$ & $128 / 5$ & $83 / 4$ & $42 / 3$ & $14 / 2$ & $75 / 4$ \\
\hline 79 & $43 / 2$ & $136 / 4$ & $267 / 9$ & $192 / 6$ & $135 / 4$ & $82 / 3$ & $37 / 2$ & $16 / 1$ & $85 / 2$ \\
\hline 80 & $37 / 2$ & $122 / 4$ & $266 / 7$ & $201 / 5$ & $134 / 3$ & $73 / 2$ & $41 / 1$ & $16 / 1$ & $73 / 2$ \\
\hline 81 & $44 / 0$ & $130 / 4$ & $267 / 9$ & $190 / 6$ & $114 / 4$ & $73 / 2$ & $35 / 1$ & $13 / 1$ & $85 / 2$ \\
\hline 82 & $41 / 1$ & $135 / 3$ & $282 / 7$ & $191 / 5$ & $138 / 3$ & $85 / 2$ & $44 / 1$ & $19 / 0$ & $82 / 1$ \\
\hline 83 & $43 / 1$ & $134 / 4$ & $263 / 8$ & $202 / 6$ & $135 / 4$ & $82 / 3$ & $46 / 1$ & $16 / 1$ & $73 / 2$ \\
\hline 84 & $36 / 0$ & $125 / 2$ & $268 / 10$ & $191 / 7$ & $127 / 5$ & $80 / 1$ & $35 / 1$ & $13 / 1$ & $84 / 1$ \\
\hline 85 & $41 / 0$ & $123 / 4$ & $271 / 12$ & $195 / 9$ & $135 / 3$ & $73 / 2$ & $36 / 1$ & $15 / 0$ & $73 / 2$ \\
\hline 86 & $43 / 0$ & $139 / 5$ & $274 / 16$ & $204 / 8$ & $124 / 6$ & $75 / 4$ & $41 / 1$ & $15 / 0$ & $86 / 3$ \\
\hline 87 & $36 / 1$ & $129 / 6$ & $288 / 13$ & $195 / 11$ & $135 / 9$ & $88 / 5$ & $44 / 2$ & $16 / 0$ & $82 / 1$ \\
\hline 88 & $48 / 0$ & $136 / 5$ & $272 / 17$ & $200 / 14$ & $140 / 8$ & $82 / 3$ & $40 / 0$ & $15 / 0$ & $75 / 4$ \\
\hline 89 & $35 / 0$ & $135 / 4$ & $279 / 21$ & $209 / 13$ & $137 / 7$ & $81 / 2$ & $41 / 1$ & $15 / 0$ & $86 / 3$ \\
\hline 90 & $38 / 2$ & $122 / 12$ & $284 / 25$ & $200 / 16$ & $132 / 9$ & $77 / 6$ & $36 / 2$ & $12 / 0$ & $77 / 6$ \\
\hline
\end{tabular}




$$
\omega=a_{0}
$$

\begin{tabular}{|c|c|c|c|c|c|c|c|c|c|}
\hline & $b_{2}$ & $b_{1}$ & $a_{0}$ & $a_{1}$ & $a_{2}$ & $a_{3}$ & $a_{4}$ & $a_{5}$ & $c_{1}$ \\
\hline 61 & $90 / 4$ & $285 / 27$ & $607 / 60$ & $432 / 42$ & $286 / 28$ & $170 / 16$ & $84 / 8$ & $31 / 0$ & $180 / 11$ \\
\hline 62 & $90 / 4$ & $297 / 22$ & $606 / 62$ & $434 / 44$ & $289 / 30$ & $172 / 18$ & $88 / 5$ & $31 / 0$ & $170 / 16$ \\
\hline 63 & $83 / 7$ & $284 / 29$ & $607 / 64$ & $436 / 46$ & $290 / 32$ & $178 / 13$ & $88 / 5$ & $31 / 0$ & $182 / 13$ \\
\hline 64 & $93 / 6$ & $289 / 31$ & $610 / 66$ & $438 / 48$ & $300 / 25$ & $178 / 13$ & $88 / 5$ & $31 / 0$ & $172 / 18$ \\
\hline 65 & $87 / 10$ & $292 / 33$ & $598 / 76$ & $436 / 46$ & $286 / 31$ & $168 / 18$ & $81 / 8$ & $27 / 1$ & $176 / 20$ \\
\hline 66 & $93 / 7$ & $289 / 31$ & $613 / 66$ & $438 / 48$ & $291 / 33$ & $174 / 20$ & $86 / 10$ & $32 / 1$ & $184 / 15$ \\
\hline 67 & $93 / 7$ & $300 / 25$ & $608 / 64$ & $436 / 46$ & $290 / 31$ & $172 / 18$ & $89 / 6$ & $32 / 1$ & $174 / 20$ \\
\hline 68 & $87 / 11$ & $287 / 32$ & $605 / 62$ & $434 / 44$ & $287 / 29$ & $178 / 13$ & $89 / 6$ & $32 / 1$ & $184 / 15$ \\
\hline 69 & $95 / 8$ & $288 / 30$ & $604 / 60$ & $432 / 42$ & $297 / 22$ & $178 / 13$ & $89 / 6$ & $32 / 1$ & $174 / 20$ \\
\hline 70 & $88 / 11$ & $287 / 28$ & $584 / 62$ & $428 / 38$ & $282 / 27$ & $168 / 18$ & $83 / 10$ & $29 / 3$ & $174 / 18$ \\
\hline 71 & $93 / 7$ & $282 / 24$ & $595 / 48$ & $426 / 36$ & $283 / 25$ & $170 / 16$ & $84 / 8$ & $33 / 2$ & $182 / 13$ \\
\hline 72 & $93 / 7$ & $294 / 19$ & $588 / 44$ & $422 / 32$ & $280 / 21$ & $166 / 12$ & $88 / 5$ & $33 / 2$ & $170 / 16$ \\
\hline 73 & $86 / 10$ & $278 / 23$ & $583 / 40$ & $418 / 28$ & $275 / 17$ & $174 / 9$ & $88 / 5$ & $33 / 2$ & $180 / 11$ \\
\hline 74 & $93 / 6$ & $277 / 19$ & $580 / 36$ & $414 / 24$ & $288 / 13$ & $174 / 9$ & $88 / 5$ & $33 / 2$ & $168 / 14$ \\
\hline 75 & $84 / 7$ & $274 / 15$ & $556 / 34$ & $412 / 22$ & $271 / 16$ & $162 / 12$ & $81 / 8$ & $30 / 4$ & $166 / 10$ \\
\hline 76 & $90 / 4$ & $271 / 13$ & $571 / 24$ & $408 / 18$ & $270 / 12$ & $162 / 8$ & $80 / 4$ & $33 / 2$ & $176 / 7$ \\
\hline 77 & $90 / 4$ & $285 / 10$ & $566 / 22$ & $406 / 16$ & $269 / 10$ & $160 / 6$ & $86 / 3$ & $33 / 2$ & $162 / 8$ \\
\hline 78 & $81 / 5$ & $266 / 11$ & $563 / 20$ & $404 / 14$ & $266 / 8$ & $170 / 5$ & $86 / 3$ & $33 / 2$ & $174 / 5$ \\
\hline 79 & $89 / 2$ & $267 / 9$ & $562 / 18$ & $402 / 12$ & $282 / 7$ & $170 / 5$ & $86 / 3$ & $33 / 2$ & $160 / 6$ \\
\hline 80 & $79 / 2$ & $266 / 7$ & $542 / 20$ & $404 / 14$ & $264 / 9$ & $156 / 6$ & $77 / 4$ & $29 / 3$ & $160 / 4$ \\
\hline 81 & $87 / 1$ & $267 / 9$ & $565 / 18$ & $402 / 12$ & $265 / 7$ & $158 / 4$ & $78 / 2$ & $32 / 1$ & $172 / 3$ \\
\hline 82 & $87 / 1$ & $282 / 7$ & $564 / 20$ & $404 / 14$ & $268 / 9$ & $160 / 6$ & $85 / 2$ & $32 / 1$ & $158 / 4$ \\
\hline 83 & $77 / 1$ & $263 / 8$ & $565 / 22$ & $406 / 16$ & $269 / 11$ & $170 / 5$ & $85 / 2$ & $32 / 1$ & $172 / 3$ \\
\hline 84 & $87 / 0$ & $268 / 10$ & $568 / 24$ & $408 / 18$ & $285 / 10$ & $170 / 5$ & $85 / 2$ & $32 / 1$ & $158 / 4$ \\
\hline 85 & $78 / 1$ & $271 / 12$ & $556 / 34$ & $412 / 22$ & $268 / 13$ & $156 / 6$ & $75 / 2$ & $27 / 1$ & $162 / 6$ \\
\hline 86 & $87 / 1$ & $274 / 16$ & $583 / 36$ & $414 / 24$ & $273 / 15$ & $162 / 8$ & $80 / 4$ & $31 / 0$ & $174 / 5$ \\
\hline 87 & $87 / 1$ & $288 / 13$ & $584 / 40$ & $418 / 28$ & $278 / 19$ & $166 / 12$ & $86 / 3$ & $31 / 0$ & $162 / 8$ \\
\hline 88 & $78 / 2$ & $272 / 17$ & $587 / 44$ & $422 / 32$ & $281 / 23$ & $174 / 9$ & $86 / 3$ & $31 / 0$ & $176 / 7$ \\
\hline 89 & $89 / 2$ & $279 / 21$ & $592 / 48$ & $426 / 36$ & $294 / 19$ & $174 / 9$ & $86 / 3$ & $31 / 0$ & $164 / 10$ \\
\hline 90 & $82 / 5$ & $284 / 25$ & $584 / 62$ & $428 / 38$ & $279 / 24$ & $162 / 12$ & $77 / 4$ & $26 / 0$ & $170 / 14$ \\
\hline
\end{tabular}




$$
\omega=a_{1}
$$

\begin{tabular}{|c|c|c|c|c|c|c|c|c|c|}
\hline & $b_{2}$ & $b_{1}$ & $a_{0}$ & $a_{1}$ & $a_{2}$ & $a_{3}$ & $\overline{a_{4}}$ & $\overline{a_{5}}$ & $c_{1}$ \\
\hline 61 & \begin{tabular}{|l|}
$66 / 3$ \\
\end{tabular} & $204 / 18$ & $432 / 42$ & $319 / 26$ & $212 / 16$ & $\begin{array}{l}126 / 9 \\
\end{array}$ & $65 / 4$ & $26 / 0$ & $128 / 9$ \\
\hline 62 & $64 / 3$ & $214 / 18$ & $434 / 44$ & $308 / 28$ & $203 / 19$ & $121 / 10$ & $61 / 2$ & $19 / 0$ & $123 / 10$ \\
\hline 63 & \begin{tabular}{|l|}
$60 / 5$ \\
\end{tabular} & $204 / 20$ & $436 / 46$ & $309 / 32$ & $207 / 21$ & $126 / 9$ & $58 / 5$ & $22 / 0$ & $130 / 11$ \\
\hline 64 & \begin{tabular}{|l|}
$68 / 5$ \\
\end{tabular} & $208 / 22$ & $438 / 48$ & $316 / 32$ & $214 / 18$ & $123 / 12$ & $65 / 4$ & $23 / 0$ & $125 / 12$ \\
\hline 65 & $65 / 4$ & $215 / 19$ & $436 / 46$ & $319 / 30$ & $207 / 23$ & $128 / 11$ & $63 / 4$ & $22 / 0$ & $130 / 11$ \\
\hline 66 & $62 / 7$ & $206 / 22$ & $438 / 48$ & $305 / 37$ & $208 / 22$ & $123 / 12$ & $59 / 6$ & $20 / 1$ & $127 / 14$ \\
\hline 67 & \begin{tabular}{|l|}
$69 / 6$ \\
\end{tabular} & $209 / 23$ & $436 / 46$ & $323 / 30$ & $215 / 19$ & $128 / 11$ & $65 / 4$ & $26 / 0$ & $130 / 11$ \\
\hline 68 & $66 / 5$ & $214 / 18$ & $434 / 44$ & $312 / 32$ & $206 / 22$ & $123 / 12$ & $64 / 5$ & $20 / 1$ & $127 / 14$ \\
\hline 69 & $63 / 8$ & $205 / 21$ & $432 / 42$ & $309 / 32$ & $206 / 20$ & $128 / 11$ & $59 / 6$ & $23 / 1$ & $130 / 11$ \\
\hline 70 & \begin{tabular}{|l|}
$68 / 5$ \\
\end{tabular} & $205 / 19$ & $428 / 38$ & $312 / 28$ & $214 / 18$ & $123 / 12$ & $66 / 5$ & $24 / 1$ & $125 / 12$ \\
\hline 71 & \begin{tabular}{|l|}
$67 / 6$ \\
\end{tabular} & $212 / 16$ & $426 / 36$ & $315 / 26$ & $202 / 18$ & $\begin{array}{l}126 / 9 \\
\end{array}$ & $63 / 4$ & $23 / 1$ & $130 / 11$ \\
\hline 72 & \begin{tabular}{|l|}
$62 / 7$ \\
\end{tabular} & $201 / 17$ & $422 / 32$ & $295 / 27$ & $202 / 16$ & $121 / 10$ & $59 / 6$ & $21 / 2$ & $123 / 10$ \\
\hline 73 & \begin{tabular}{|l|}
$67 / 4$ \\
\end{tabular} & $200 / 14$ & $418 / 28$ & $313 / 20$ & $209 / 13$ & $\begin{array}{c}126 / 9 \\
\end{array}$ & $65 / 4$ & $27 / 1$ & $\begin{array}{l}128 / 9 \\
\end{array}$ \\
\hline 74 & $66 / 5$ & $207 / 11$ & $414 / 24$ & $300 / 20$ & $198 / 14$ & $\begin{array}{l}119 / 8 \\
\end{array}$ & $63 / 4$ & $21 / 2$ & $121 / 8$ \\
\hline 75 & $60 / 5$ & $196 / 12$ & $412 / 22$ & $295 / 18$ & $197 / 11$ & $124 / 7$ & $58 / 5$ & $24 / 2$ & $126 / 7$ \\
\hline 76 & $66 / 3$ & $196 / 10$ & $408 / 18$ & $298 / 14$ & $204 / 8$ & $117 / 6$ & $63 / 2$ & $24 / 1$ & $119 / 6$ \\
\hline 77 & \begin{tabular}{|l|l|}
$64 / 3$ \\
\end{tabular} & $202 / 6$ & $406 / 16$ & $301 / 12$ & $193 / 9$ & $\begin{array}{l}122 / 5 \\
\end{array}$ & $63 / 4$ & $24 / 2$ & $124 / 5$ \\
\hline 78 & \begin{tabular}{|l|}
$58 / 3$ \\
\end{tabular} & $192 / 8$ & $404 / 14$ & $281 / 13$ & $193 / 7$ & $117 / 6$ & $56 / 3$ & $21 / 2$ & $117 / 4$ \\
\hline 79 & \begin{tabular}{|l|}
$64 / 1$ \\
\end{tabular} & $192 / 6$ & $402 / 12$ & $301 / 8$ & $202 / 6$ & $120 / 3$ & $63 / 2$ & $27 / 1$ & $122 / 3$ \\
\hline 80 & $63 / 2$ & $201 / 5$ & $404 / 14$ & $290 / 10$ & $189 / 5$ & $115 / 4$ & $61 / 2$ & $21 / 2$ & $117 / 4$ \\
\hline 81 & 56 & $190 / 6$ & $402 / 12$ & $285 / 8$ & $192 / 6$ & $120 / 3$ & $55 / 2$ & $23 / 1$ & $120 / 1$ \\
\hline 82 & \begin{tabular}{|l|}
$63 / 0$ \\
\end{tabular} & $191 / 5$ & $404 / 14$ & $294 / 10$ & $201 / 5$ & $115 / 4$ & $63 / 2$ & $24 / 1$ & $117 / 4$ \\
\hline 83 & \begin{tabular}{|l|}
$62 / 1$ \\
\end{tabular} & $202 / 6$ & $406 / 16$ & $297 / 8$ & $190 / 6$ & $\begin{array}{l}120 / 3 \\
\end{array}$ & $60 / 1$ & $23 / 1$ & $120 / 1$ \\
\hline 84 & \begin{tabular}{|l|l}
$55 / 0$ \\
\end{tabular} & $\begin{array}{l}191 / 7 \\
\end{array}$ & $408 / 18$ & $281 / 13$ & $194 / 8$ & $115 / 4$ & $55 / 2$ & $20 / 1$ & $117 / 4$ \\
\hline 85 & \begin{tabular}{|l|}
$64 / 1$ \\
\end{tabular} & $195 / 9$ & $412 / 22$ & $305 / 12$ & $202 / 6$ & $120 / 3$ & $62 / 1$ & $26 / 0$ & $122 / 3$ \\
\hline 86 & $61 / 0$ & $204 / 8$ & $414 / 24$ & $294 / 14$ & $194 / 10$ & $117 / 6$ & $61 / 2$ & $20 / 1$ & $117 / 4$ \\
\hline 87 & 56 & $195 / 11$ & $418 / 28$ & $295 / 18$ & $198 / 12$ & $122 / 5$ & $55 / 2$ & $22 / 0$ & $124 / 5$ \\
\hline 88 & \begin{tabular}{|l|}
$65 / 2$ \\
\end{tabular} & $200 / 14$ & $422 / 32$ & $304 / 20$ & $207 / 11$ & $\begin{array}{l}117 / 6 \\
\end{array}$ & $63 / 2$ & $23 / 0$ & $119 / 6$ \\
\hline 89 & \begin{tabular}{|l|}
$62 / 1$ \\
\end{tabular} & $209 / 13$ & $426 / 36$ & $309 / 20$ & $198 / 14$ & $124 / 7$ & $61 / 2$ & $22 / 0$ & $126 / 7$ \\
\hline 90 & 58 & $200 / 16$ & $428 / 38$ & $295 / 27$ & $203 / 17$ & $119 / 8$ & $56 / 3$ & $19 / 0$ & $121 / 8$ \\
\hline
\end{tabular}




$$
\omega=a_{2}
$$

\begin{tabular}{|c||c|c|c|c|c|c|c|c|c|}
\hline & $b_{2}$ & $b_{1}$ & $a_{0}$ & $a_{1}$ & $a_{2}$ & $a_{3}$ & $a_{4}$ & $a_{5}$ & $c_{1}$ \\
\hline \hline 61 & $43 / 2$ & $133 / 14$ & $286 / 28$ & $212 / 16$ & $146 / 11$ & $89 / 6$ & $46 / 3$ & $19 / 0$ & $88 / 5$ \\
\hline 62 & $45 / 2$ & $146 / 12$ & $289 / 30$ & $203 / 19$ & $141 / 10$ & $84 / 5$ & $45 / 0$ & $15 / 0$ & $79 / 8$ \\
\hline 63 & $39 / 4$ & $136 / 13$ & $290 / 32$ & $207 / 21$ & $134 / 12$ & $82 / 3$ & $36 / 2$ & $12 / 0$ & $90 / 7$ \\
\hline 64 & $49 / 1$ & $142 / 11$ & $300 / 25$ & $214 / 18$ & $142 / 10$ & $79 / 8$ & $39 / 4$ & $15 / 0$ & $86 / 5$ \\
\hline 65 & $39 / 4$ & $143 / 12$ & $286 / 31$ & $207 / 23$ & $134 / 16$ & $81 / 10$ & $43 / 3$ & $15 / 0$ & $81 / 10$ \\
\hline 66 & $42 / 6$ & $130 / 20$ & $291 / 33$ & $208 / 22$ & $142 / 16$ & $90 / 7$ & $45 / 3$ & $16 / 0$ & $90 / 7$ \\
\hline 67 & $51 / 2$ & $147 / 12$ & $290 / 31$ & $215 / 19$ & $143 / 11$ & $84 / 5$ & $41 / 1$ & $15 / 0$ & $81 / 10$ \\
\hline 68 & $39 / 5$ & $142 / 11$ & $287 / 29$ & $206 / 22$ & $142 / 12$ & $85 / 6$ & $43 / 3$ & $15 / 0$ & $90 / 7$ \\
\hline 69 & $46 / 3$ & $135 / 13$ & $297 / 22$ & $206 / 20$ & $137 / 14$ & $81 / 10$ & $39 / 5$ & $13 / 1$ & $87 / 6$ \\
\hline 70 & $45 / 4$ & $144 / 12$ & $282 / 27$ & $214 / 18$ & $135 / 16$ & $81 / 10$ & $40 / 5$ & $16 / 1$ & $81 / 10$ \\
\hline 71 & $41 / 6$ & $132 / 14$ & $283 / 25$ & $202 / 18$ & $145 / 11$ & $88 / 5$ & $49 / 1$ & $19 / 0$ & $89 / 6$ \\
\hline 72 & $46 / 2$ & $135 / 9$ & $280 / 21$ & $202 / 16$ & $133 / 10$ & $83 / 4$ & $41 / 2$ & $13 / 1$ & $79 / 8$ \\
\hline 73 & $43 / 3$ & $139 / 7$ & $275 / 17$ & $209 / 13$ & $140 / 9$ & $86 / 7$ & $40 / 5$ & $16 / 1$ & $87 / 4$ \\
\hline 74 & $45 / 3$ & $138 / 8$ & $288 / 13$ & $198 / 14$ & $141 / 10$ & $79 / 8$ & $43 / 3$ & $16 / 1$ & $86 / 5$ \\
\hline 75 & $40 / 4$ & $132 / 9$ & $271 / 16$ & $197 / 11$ & $122 / 12$ & $77 / 6$ & $38 / 4$ & $14 / 2$ & $77 / 6$ \\
\hline 76 & $43 / 2$ & $125 / 6$ & $270 / 12$ & $204 / 8$ & $139 / 4$ & $85 / 2$ & $43 / 0$ & $19 / 0$ & $86 / 3$ \\
\hline 77 & $44 / 1$ & $137 / 3$ & $269 / 10$ & $193 / 9$ & $136 / 5$ & $82 / 3$ & $47 / 2$ & $16 / 1$ & $75 / 4$ \\
\hline 78 & $37 / 2$ & $128 / 5$ & $266 / 8$ & $193 / 7$ & $128 / 6$ & $84 / 5$ & $38 / 4$ & $14 / 2$ & $84 / 1$ \\
\hline 79 & $49 / 1$ & $135 / 4$ & $282 / 7$ & $202 / 6$ & $137 / 5$ & $75 / 4$ & $37 / 2$ & $16 / 1$ & $84 / 3$ \\
\hline 80 & $37 / 2$ & $134 / 3$ & $264 / 9$ & $189 / 5$ & $122 / 4$ & $73 / 2$ & $41 / 1$ & $16 / 1$ & $73 / 2$ \\
\hline 81 & $36 / 0$ & $114 / 4$ & $265 / 7$ & $192 / 6$ & $128 / 2$ & $84 / 1$ & $42 / 0$ & $16 / 0$ & $84 / 1$ \\
\hline 82 & $49 / 0$ & $138 / 3$ & $268 / 9$ & $201 / 5$ & $136 / 4$ & $82 / 3$ & $42 / 2$ & $16 / 1$ & $73 / 2$ \\
\hline 83 & $35 / 1$ & $135 / 4$ & $269 / 11$ & $190 / 6$ & $133 / 3$ & $81 / 2$ & $41 / 1$ & $16 / 1$ & $84 / 1$ \\
\hline 84 & $43 / 0$ & $127 / 5$ & $285 / 10$ & $194 / 8$ & $127 / 4$ & $73 / 2$ & $35 / 1$ & $13 / 1$ & $83 / 2$ \\
\hline 85 & $41 / 0$ & $135 / 3$ & $268 / 13$ & $202 / 6$ & $123 / 4$ & $73 / 2$ & $36 / 1$ & $15 / 0$ & $73 / 2$ \\
\hline 86 & $35 / 0$ & $124 / 6$ & $273 / 15$ & $194 / 10$ & $138 / 4$ & $86 / 3$ & $49 / 1$ & $19 / 0$ & $85 / 2$ \\
\hline 87 & $45 / 1$ & $135 / 9$ & $278 / 19$ & $198 / 12$ & $131 / 8$ & $83 / 4$ & $40 / 1$ & $13 / 1$ & $75 / 4$ \\
\hline 88 & $41 / 1$ & $140 / 8$ & $281 / 23$ & $207 / 11$ & $137 / 6$ & $80 / 1$ & $36 / 1$ & $15 / 0$ & $87 / 4$ \\
\hline 89 & $42 / 0$ & $137 / 7$ & $294 / 19$ & $198 / 14$ & $136 / 5$ & $75 / 4$ & $41 / 1$ & $15 / 0$ & $84 / 3$ \\
\hline 90 & $38 / 2$ & $132 / 9$ & $279 / 24$ & $203 / 17$ & $122 / 12$ & $77 / 6$ & $36 / 2$ & $12 / 0$ & $77 / 6$ \\
\hline
\end{tabular}




$$
\omega=a_{3}
$$

\begin{tabular}{|c||c|c|c|c|c|c|c|c|c|}
\hline & $b_{2}$ & $b_{1}$ & $a_{0}$ & $a_{1}$ & $a_{2}$ & $a_{3}$ & $a_{4}$ & $a_{5}$ & $c_{1}$ \\
\hline \hline 61 & $27 / 1$ & $79 / 8$ & $170 / 16$ & $126 / 9$ & $89 / 6$ & $53 / 4$ & $28 / 2$ & $12 / 0$ & $53 / 4$ \\
\hline 62 & $28 / 2$ & $90 / 7$ & $172 / 18$ & $121 / 10$ & $84 / 5$ & $55 / 3$ & $30 / 0$ & $10 / 0$ & $47 / 4$ \\
\hline 63 & $24 / 0$ & $83 / 4$ & $178 / 13$ & $126 / 9$ & $82 / 3$ & $52 / 0$ & $24 / 0$ & $9 / 0$ & $58 / 2$ \\
\hline 64 & $27 / 1$ & $84 / 5$ & $178 / 13$ & $123 / 12$ & $79 / 8$ & $45 / 2$ & $21 / 1$ & $7 / 0$ & $47 / 4$ \\
\hline 65 & $23 / 3$ & $81 / 10$ & $168 / 18$ & $128 / 11$ & $81 / 10$ & $46 / 6$ & $23 / 3$ & $9 / 0$ & $51 / 4$ \\
\hline 66 & $28 / 2$ & $81 / 10$ & $174 / 20$ & $123 / 12$ & $90 / 7$ & $51 / 6$ & $28 / 2$ & $10 / 0$ & $51 / 6$ \\
\hline 67 & $28 / 2$ & $89 / 6$ & $172 / 18$ & $128 / 11$ & $84 / 5$ & $58 / 2$ & $30 / 0$ & $12 / 0$ & $51 / 4$ \\
\hline 68 & $25 / 1$ & $84 / 5$ & $178 / 13$ & $123 / 12$ & $85 / 6$ & $49 / 1$ & $24 / 0$ & $7 / 0$ & $55 / 3$ \\
\hline 69 & $28 / 2$ & $86 / 7$ & $178 / 13$ & $128 / 11$ & $81 / 10$ & $51 / 4$ & $23 / 3$ & $9 / 0$ & $51 / 4$ \\
\hline 70 & $24 / 4$ & $81 / 10$ & $168 / 18$ & $123 / 12$ & $81 / 10$ & $45 / 9$ & $24 / 4$ & $8 / 1$ & $49 / 6$ \\
\hline 71 & $28 / 2$ & $79 / 8$ & $170 / 16$ & $126 / 9$ & $88 / 5$ & $53 / 4$ & $27 / 1$ & $12 / 0$ & $53 / 4$ \\
\hline 72 & $27 / 1$ & $86 / 3$ & $166 / 12$ & $121 / 10$ & $83 / 4$ & $53 / 1$ & $30 / 0$ & $10 / 0$ & $47 / 4$ \\
\hline 73 & $26 / 2$ & $84 / 5$ & $174 / 9$ & $126 / 9$ & $86 / 7$ & $54 / 2$ & $25 / 1$ & $9 / 0$ & $58 / 2$ \\
\hline 74 & $28 / 2$ & $85 / 6$ & $174 / 9$ & $119 / 8$ & $79 / 8$ & $49 / 6$ & $24 / 4$ & $8 / 1$ & $47 / 4$ \\
\hline 75 & $23 / 3$ & $77 / 6$ & $162 / 12$ & $124 / 7$ & $77 / 6$ & $46 / 6$ & $23 / 3$ & $10 / 1$ & $51 / 4$ \\
\hline 76 & $27 / 1$ & $75 / 4$ & $162 / 8$ & $117 / 6$ & $85 / 2$ & $47 / 2$ & $26 / 0$ & $10 / 0$ & $47 / 2$ \\
\hline 77 & $26 / 0$ & $84 / 1$ & $160 / 6$ & $122 / 5$ & $82 / 3$ & $56 / 0$ & $30 / 0$ & $12 / 0$ & $49 / 2$ \\
\hline 78 & $26 / 2$ & $83 / 4$ & $170 / 5$ & $117 / 6$ & $84 / 5$ & $51 / 3$ & $26 / 2$ & $8 / 1$ & $53 / 1$ \\
\hline 79 & $27 / 1$ & $82 / 3$ & $170 / 5$ & $120 / 3$ & $75 / 4$ & $51 / 4$ & $23 / 3$ & $10 / 1$ & $49 / 2$ \\
\hline 80 & $21 / 1$ & $73 / 2$ & $156 / 6$ & $115 / 4$ & $73 / 2$ & $39 / 3$ & $21 / 1$ & $8 / 1$ & $45 / 2$ \\
\hline 81 & $26 / 0$ & $73 / 2$ & $158 / 4$ & $120 / 3$ & $84 / 1$ & $49 / 0$ & $26 / 0$ & $12 / 0$ & $49 / 0$ \\
\hline 82 & $26 / 0$ & $85 / 2$ & $160 / 6$ & $115 / 4$ & $82 / 3$ & $53 / 1$ & $30 / 0$ & $10 / 0$ & $45 / 2$ \\
\hline 83 & $25 / 1$ & $82 / 3$ & $170 / 5$ & $120 / 3$ & $81 / 2$ & $54 / 2$ & $26 / 2$ & $10 / 1$ & $56 / 0$ \\
\hline 84 & $26 / 0$ & $80 / 1$ & $170 / 5$ & $115 / 4$ & $73 / 2$ & $45 / 2$ & $21 / 1$ & $8 / 1$ & $45 / 2$ \\
\hline 85 & $20 / 0$ & $73 / 2$ & $156 / 6$ & $120 / 3$ & $73 / 2$ & $40 / 0$ & $20 / 0$ & $9 / 0$ & $47 / 0$ \\
\hline 86 & $26 / 0$ & $75 / 4$ & $162 / 8$ & $117 / 6$ & $86 / 3$ & $47 / 2$ & $27 / 1$ & $10 / 0$ & $47 / 2$ \\
\hline 87 & $27 / 1$ & $88 / 5$ & $166 / 12$ & $122 / 5$ & $83 / 4$ & $58 / 2$ & $30 / 0$ & $12 / 0$ & $49 / 2$ \\
\hline 88 & $24 / 0$ & $82 / 3$ & $174 / 9$ & $117 / 6$ & $80 / 1$ & $49 / 1$ & $25 / 1$ & $8 / 1$ & $53 / 1$ \\
\hline 89 & $26 / 0$ & $81 / 2$ & $174 / 9$ & $124 / 7$ & $75 / 4$ & $47 / 0$ & $20 / 0$ & $9 / 0$ & $49 / 2$ \\
\hline 90 & $21 / 1$ & $77 / 6$ & $162 / 12$ & $119 / 8$ & $77 / 6$ & $39 / 3$ & $21 / 1$ & $7 / 0$ & $45 / 2$ \\
\hline
\end{tabular}




$$
\omega=a_{4}
$$

\begin{tabular}{|c||c|c|c|c|c|c|c|c|c|}
\hline & $b_{2}$ & $b_{1}$ & $a_{0}$ & $a_{1}$ & $a_{2}$ & $a_{3}$ & $a_{4}$ & $a_{5}$ & $c_{1}$ \\
\hline \hline 61 & $15 / 1$ & $39 / 4$ & $84 / 8$ & $65 / 4$ & $46 / 3$ & $28 / 2$ & $15 / 1$ & $7 / 0$ & $28 / 2$ \\
\hline 62 & $16 / 0$ & $50 / 2$ & $88 / 5$ & $61 / 2$ & $45 / 0$ & $30 / 0$ & $16 / 0$ & $5 / 0$ & $24 / 0$ \\
\hline 63 & $9 / 0$ & $39 / 0$ & $88 / 5$ & $58 / 5$ & $36 / 2$ & $24 / 0$ & $13 / 0$ & $5 / 0$ & $28 / 2$ \\
\hline 64 & $15 / 1$ & $39 / 4$ & $88 / 5$ & $65 / 4$ & $39 / 4$ & $21 / 1$ & $13 / 0$ & $5 / 0$ & $24 / 0$ \\
\hline 65 & $12 / 0$ & $43 / 3$ & $81 / 8$ & $63 / 4$ & $43 / 3$ & $23 / 3$ & $9 / 0$ & $3 / 0$ & $23 / 3$ \\
\hline 66 & $12 / 2$ & $39 / 5$ & $86 / 10$ & $59 / 6$ & $45 / 3$ & $28 / 2$ & $12 / 2$ & $5 / 0$ & $28 / 2$ \\
\hline 67 & $17 / 0$ & $45 / 2$ & $89 / 6$ & $65 / 4$ & $41 / 1$ & $30 / 0$ & $17 / 0$ & $7 / 0$ & $25 / 1$ \\
\hline 68 & $12 / 0$ & $46 / 1$ & $89 / 6$ & $64 / 5$ & $43 / 3$ & $24 / 0$ & $16 / 0$ & $5 / 0$ & $28 / 2$ \\
\hline 69 & $12 / 2$ & $39 / 5$ & $89 / 6$ & $59 / 6$ & $39 / 5$ & $23 / 3$ & $9 / 0$ & $3 / 0$ & $25 / 1$ \\
\hline 70 & $14 / 1$ & $40 / 5$ & $83 / 10$ & $66 / 5$ & $40 / 5$ & $24 / 4$ & $12 / 2$ & $5 / 0$ & $24 / 4$ \\
\hline 71 & $14 / 1$ & $43 / 3$ & $84 / 8$ & $63 / 4$ & $49 / 1$ & $27 / 1$ & $14 / 1$ & $5 / 0$ & $27 / 1$ \\
\hline 72 & $13 / 0$ & $43 / 1$ & $88 / 5$ & $59 / 6$ & $41 / 2$ & $30 / 0$ & $13 / 0$ & $5 / 0$ & $26 / 2$ \\
\hline 73 & $14 / 1$ & $43 / 3$ & $88 / 5$ & $65 / 4$ & $40 / 5$ & $25 / 1$ & $17 / 0$ & $7 / 0$ & $27 / 1$ \\
\hline 74 & $14 / 1$ & $43 / 3$ & $88 / 5$ & $63 / 4$ & $43 / 3$ & $24 / 4$ & $12 / 0$ & $3 / 0$ & $26 / 2$ \\
\hline 75 & $11 / 2$ & $38 / 4$ & $81 / 8$ & $58 / 5$ & $38 / 4$ & $23 / 3$ & $10 / 4$ & $4 / 1$ & $23 / 3$ \\
\hline 76 & $14 / 0$ & $37 / 2$ & $80 / 4$ & $63 / 2$ & $43 / 0$ & $26 / 0$ & $14 / 0$ & $7 / 0$ & $26 / 0$ \\
\hline 77 & $16 / 0$ & $48 / 0$ & $86 / 3$ & $63 / 4$ & $47 / 2$ & $30 / 0$ & $16 / 0$ & $5 / 0$ & $26 / 2$ \\
\hline 78 & $11 / 2$ & $42 / 3$ & $86 / 3$ & $56 / 3$ & $38 / 4$ & $26 / 2$ & $13 / 0$ & $5 / 0$ & $26 / 0$ \\
\hline 79 & $14 / 0$ & $37 / 2$ & $86 / 3$ & $63 / 2$ & $37 / 2$ & $23 / 3$ & $14 / 1$ & $5 / 0$ & $26 / 2$ \\
\hline 80 & $13 / 1$ & $41 / 1$ & $77 / 4$ & $61 / 2$ & $41 / 1$ & $21 / 1$ & $11 / 2$ & $4 / 1$ & $21 / 1$ \\
\hline 81 & $10 / 0$ & $35 / 1$ & $78 / 2$ & $55 / 2$ & $42 / 0$ & $26 / 0$ & $10 / 0$ & $5 / 0$ & $26 / 0$ \\
\hline 82 & $17 / 0$ & $44 / 1$ & $85 / 2$ & $63 / 2$ & $42 / 2$ & $30 / 0$ & $17 / 0$ & $7 / 0$ & $25 / 1$ \\
\hline 83 & $13 / 1$ & $46 / 1$ & $85 / 2$ & $60 / 1$ & $41 / 1$ & $26 / 2$ & $16 / 0$ & $5 / 0$ & $26 / 0$ \\
\hline 84 & $10 / 0$ & $35 / 1$ & $85 / 2$ & $55 / 2$ & $35 / 1$ & $21 / 1$ & $11 / 2$ & $4 / 1$ & $25 / 1$ \\
\hline 85 & $13 / 0$ & $36 / 1$ & $75 / 2$ & $62 / 1$ & $36 / 1$ & $20 / 0$ & $10 / 0$ & $5 / 0$ & $20 / 0$ \\
\hline 86 & $13 / 0$ & $41 / 1$ & $80 / 4$ & $61 / 2$ & $49 / 1$ & $27 / 1$ & $13 / 0$ & $5 / 0$ & $27 / 1$ \\
\hline 87 & $13 / 0$ & $44 / 2$ & $86 / 3$ & $55 / 2$ & $40 / 1$ & $30 / 0$ & $13 / 0$ & $5 / 0$ & $24 / 0$ \\
\hline 88 & $13 / 0$ & $40 / 0$ & $86 / 3$ & $63 / 2$ & $36 / 1$ & $25 / 1$ & $17 / 0$ & $7 / 0$ & $27 / 1$ \\
\hline 89 & $13 / 0$ & $41 / 1$ & $86 / 3$ & $61 / 2$ & $41 / 1$ & $20 / 0$ & $13 / 1$ & $4 / 1$ & $24 / 0$ \\
\hline 90 & $9 / 0$ & $36 / 2$ & $77 / 4$ & $56 / 3$ & $36 / 2$ & $21 / 1$ & $6 / 0$ & $3 / 0$ & $21 / 1$ \\
\hline
\end{tabular}




$$
\omega=a_{5}
$$

\begin{tabular}{|c||c|c|c|c|c|c|c|c|c|}
\hline & $b_{2}$ & $b_{1}$ & $a_{0}$ & $a_{1}$ & $a_{2}$ & $a_{3}$ & $a_{4}$ & $a_{5}$ & $c_{1}$ \\
\hline \hline 61 & $7 / 0$ & $15 / 0$ & $31 / 0$ & $26 / 0$ & $19 / 0$ & $12 / 0$ & $7 / 0$ & $3 / 0$ & $12 / 0$ \\
\hline 62 & $5 / 0$ & $19 / 0$ & $31 / 0$ & $19 / 0$ & $15 / 0$ & $10 / 0$ & $5 / 0$ & $2 / 0$ & $7 / 0$ \\
\hline 63 & $3 / 0$ & $12 / 0$ & $31 / 0$ & $22 / 0$ & $12 / 0$ & $9 / 0$ & $5 / 0$ & $2 / 0$ & $12 / 0$ \\
\hline 64 & $7 / 0$ & $15 / 0$ & $31 / 0$ & $23 / 0$ & $15 / 0$ & $7 / 0$ & $5 / 0$ & $2 / 0$ & $7 / 0$ \\
\hline 65 & $3 / 0$ & $15 / 0$ & $27 / 1$ & $22 / 0$ & $15 / 0$ & $9 / 0$ & $3 / 0$ & $2 / 0$ & $9 / 0$ \\
\hline 66 & $5 / 0$ & $13 / 1$ & $32 / 1$ & $20 / 1$ & $16 / 0$ & $10 / 0$ & $5 / 0$ & $1 / 0$ & $10 / 0$ \\
\hline 67 & $7 / 0$ & $19 / 0$ & $32 / 1$ & $26 / 0$ & $15 / 0$ & $12 / 0$ & $7 / 0$ & $3 / 0$ & $9 / 0$ \\
\hline 68 & $3 / 0$ & $15 / 0$ & $32 / 1$ & $20 / 1$ & $15 / 0$ & $7 / 0$ & $5 / 0$ & $2 / 0$ & $10 / 0$ \\
\hline 69 & $5 / 0$ & $13 / 1$ & $32 / 1$ & $23 / 1$ & $13 / 1$ & $9 / 0$ & $3 / 0$ & $2 / 0$ & $9 / 0$ \\
\hline 70 & $5 / 0$ & $16 / 1$ & $29 / 3$ & $24 / 1$ & $16 / 1$ & $8 / 1$ & $5 / 0$ & $1 / 0$ & $8 / 1$ \\
\hline 71 & $5 / 0$ & $16 / 1$ & $33 / 2$ & $23 / 1$ & $19 / 0$ & $12 / 0$ & $5 / 0$ & $3 / 0$ & $12 / 0$ \\
\hline 72 & $5 / 0$ & $16 / 0$ & $33 / 2$ & $21 / 2$ & $13 / 1$ & $10 / 0$ & $5 / 0$ & $1 / 0$ & $8 / 1$ \\
\hline 73 & $5 / 0$ & $16 / 1$ & $33 / 2$ & $27 / 1$ & $16 / 1$ & $9 / 0$ & $7 / 0$ & $3 / 0$ & $12 / 0$ \\
\hline 74 & $5 / 0$ & $16 / 1$ & $33 / 2$ & $21 / 2$ & $16 / 1$ & $8 / 1$ & $3 / 0$ & $2 / 0$ & $8 / 1$ \\
\hline 75 & $4 / 1$ & $14 / 2$ & $30 / 4$ & $24 / 2$ & $14 / 2$ & $10 / 1$ & $4 / 1$ & $1 / 0$ & $10 / 1$ \\
\hline 76 & $7 / 0$ & $16 / 1$ & $33 / 2$ & $24 / 1$ & $19 / 0$ & $10 / 0$ & $7 / 0$ & $2 / 0$ & $10 / 0$ \\
\hline 77 & $5 / 0$ & $19 / 0$ & $33 / 2$ & $24 / 2$ & $16 / 1$ & $12 / 0$ & $5 / 0$ & $3 / 0$ & $10 / 1$ \\
\hline 78 & $4 / 1$ & $14 / 2$ & $33 / 2$ & $21 / 2$ & $14 / 2$ & $8 / 1$ & $5 / 0$ & $1 / 0$ & $10 / 0$ \\
\hline 79 & $7 / 0$ & $16 / 1$ & $33 / 2$ & $27 / 1$ & $16 / 1$ & $10 / 1$ & $5 / 0$ & $3 / 0$ & $10 / 1$ \\
\hline 80 & $4 / 1$ & $16 / 1$ & $29 / 3$ & $21 / 2$ & $16 / 1$ & $8 / 1$ & $4 / 1$ & $1 / 0$ & $8 / 1$ \\
\hline 81 & $5 / 0$ & $13 / 1$ & $32 / 1$ & $23 / 1$ & $16 / 0$ & $12 / 0$ & $5 / 0$ & $2 / 0$ & $12 / 0$ \\
\hline 82 & $7 / 0$ & $19 / 0$ & $32 / 1$ & $24 / 1$ & $16 / 1$ & $10 / 0$ & $7 / 0$ & $2 / 0$ & $8 / 1$ \\
\hline 83 & $4 / 1$ & $16 / 1$ & $32 / 1$ & $23 / 1$ & $16 / 1$ & $10 / 1$ & $5 / 0$ & $3 / 0$ & $12 / 0$ \\
\hline 84 & $5 / 0$ & $13 / 1$ & $32 / 1$ & $20 / 1$ & $13 / 1$ & $8 / 1$ & $4 / 1$ & $1 / 0$ & $8 / 1$ \\
\hline 85 & $5 / 0$ & $15 / 0$ & $27 / 1$ & $26 / 0$ & $15 / 0$ & $9 / 0$ & $5 / 0$ & $2 / 0$ & $9 / 0$ \\
\hline 86 & $5 / 0$ & $15 / 0$ & $31 / 0$ & $20 / 1$ & $19 / 0$ & $10 / 0$ & $5 / 0$ & $2 / 0$ & $10 / 0$ \\
\hline 87 & $5 / 0$ & $16 / 0$ & $31 / 0$ & $22 / 0$ & $13 / 1$ & $12 / 0$ & $5 / 0$ & $2 / 0$ & $9 / 0$ \\
\hline 88 & $5 / 0$ & $15 / 0$ & $31 / 0$ & $23 / 0$ & $15 / 0$ & $8 / 1$ & $7 / 0$ & $2 / 0$ & $10 / 0$ \\
\hline 89 & $5 / 0$ & $15 / 0$ & $31 / 0$ & $22 / 0$ & $15 / 0$ & $9 / 0$ & $4 / 1$ & $3 / 0$ & $9 / 0$ \\
\hline 90 & $3 / 0$ & $12 / 0$ & $26 / 0$ & $19 / 0$ & $12 / 0$ & $7 / 0$ & $3 / 0$ & $1 / 1$ & $7 / 0$ \\
\hline
\end{tabular}




$$
\omega=c_{1}
$$

\begin{tabular}{|c||c|c|c|c|c|c|c|c|c|}
\hline & $b_{2}$ & $b_{1}$ & $a_{0}$ & $a_{1}$ & $a_{2}$ & $a_{3}$ & $a_{4}$ & $a_{5}$ & $c_{1}$ \\
\hline \hline 61 & $32 / 0$ & $88 / 5$ & $180 / 11$ & $128 / 9$ & $88 / 5$ & $53 / 4$ & $28 / 2$ & $12 / 0$ & $60 / 2$ \\
\hline 62 & $21 / 1$ & $85 / 4$ & $170 / 16$ & $123 / 10$ & $79 / 8$ & $47 / 4$ & $24 / 0$ & $7 / 0$ & $45 / 2$ \\
\hline 63 & $27 / 1$ & $79 / 8$ & $182 / 13$ & $130 / 11$ & $90 / 7$ & $58 / 2$ & $28 / 2$ & $12 / 0$ & $53 / 4$ \\
\hline 64 & $27 / 1$ & $89 / 6$ & $172 / 18$ & $125 / 12$ & $86 / 5$ & $47 / 4$ & $24 / 0$ & $7 / 0$ & $53 / 1$ \\
\hline 65 & $23 / 3$ & $81 / 10$ & $176 / 20$ & $130 / 11$ & $81 / 10$ & $51 / 4$ & $23 / 3$ & $9 / 0$ & $46 / 6$ \\
\hline 66 & $32 / 0$ & $89 / 6$ & $184 / 15$ & $127 / 14$ & $90 / 7$ & $51 / 6$ & $28 / 2$ & $10 / 0$ & $57 / 3$ \\
\hline 67 & $23 / 3$ & $88 / 7$ & $174 / 20$ & $130 / 11$ & $81 / 10$ & $51 / 4$ & $25 / 1$ & $9 / 0$ & $51 / 4$ \\
\hline 68 & $28 / 2$ & $81 / 10$ & $184 / 15$ & $127 / 14$ & $90 / 7$ & $55 / 3$ & $28 / 2$ & $10 / 0$ & $51 / 6$ \\
\hline 69 & $28 / 2$ & $90 / 7$ & $174 / 20$ & $130 / 11$ & $87 / 6$ & $51 / 4$ & $25 / 1$ & $9 / 0$ & $58 / 2$ \\
\hline 70 & $24 / 4$ & $81 / 10$ & $174 / 18$ & $125 / 12$ & $81 / 10$ & $49 / 6$ & $24 / 4$ & $8 / 1$ & $45 / 9$ \\
\hline 71 & $32 / 0$ & $88 / 5$ & $182 / 13$ & $130 / 11$ & $89 / 6$ & $53 / 4$ & $27 / 1$ & $12 / 0$ & $60 / 2$ \\
\hline 72 & $24 / 4$ & $88 / 7$ & $170 / 16$ & $123 / 10$ & $79 / 8$ & $47 / 4$ & $26 / 2$ & $8 / 1$ & $49 / 6$ \\
\hline 73 & $28 / 2$ & $79 / 8$ & $180 / 11$ & $128 / 9$ & $87 / 4$ & $58 / 2$ & $27 / 1$ & $12 / 0$ & $53 / 4$ \\
\hline 74 & $28 / 2$ & $88 / 5$ & $168 / 14$ & $121 / 8$ & $86 / 5$ & $47 / 4$ & $26 / 2$ & $8 / 1$ & $55 / 3$ \\
\hline 75 & $23 / 3$ & $77 / 6$ & $166 / 10$ & $126 / 7$ & $77 / 6$ & $51 / 4$ & $23 / 3$ & $10 / 1$ & $46 / 6$ \\
\hline 76 & $32 / 0$ & $86 / 3$ & $176 / 7$ & $119 / 6$ & $86 / 3$ & $47 / 2$ & $26 / 0$ & $10 / 0$ & $55 / 1$ \\
\hline 77 & $23 / 3$ & $85 / 4$ & $162 / 8$ & $124 / 5$ & $75 / 4$ & $49 / 2$ & $26 / 2$ & $10 / 1$ & $51 / 4$ \\
\hline 78 & $27 / 1$ & $75 / 4$ & $174 / 5$ & $117 / 4$ & $84 / 1$ & $53 / 1$ & $26 / 0$ & $10 / 0$ & $47 / 2$ \\
\hline 79 & $27 / 1$ & $85 / 2$ & $160 / 6$ & $122 / 3$ & $84 / 3$ & $49 / 2$ & $26 / 2$ & $10 / 1$ & $58 / 2$ \\
\hline 80 & $21 / 1$ & $73 / 2$ & $160 / 4$ & $117 / 4$ & $73 / 2$ & $45 / 2$ & $21 / 1$ & $8 / 1$ & $39 / 3$ \\
\hline 81 & $32 / 0$ & $85 / 2$ & $172 / 3$ & $120 / 1$ & $84 / 1$ & $49 / 0$ & $26 / 0$ & $12 / 0$ & $58 / 0$ \\
\hline 82 & $21 / 1$ & $82 / 1$ & $158 / 4$ & $117 / 4$ & $73 / 2$ & $45 / 2$ & $25 / 1$ & $8 / 1$ & $45 / 2$ \\
\hline 83 & $26 / 0$ & $73 / 2$ & $172 / 3$ & $120 / 1$ & $84 / 1$ & $56 / 0$ & $26 / 0$ & $12 / 0$ & $49 / 0$ \\
\hline 84 & $26 / 0$ & $84 / 1$ & $158 / 4$ & $117 / 4$ & $83 / 2$ & $45 / 2$ & $25 / 1$ & $8 / 1$ & $53 / 1$ \\
\hline 85 & $20 / 0$ & $73 / 2$ & $162 / 6$ & $122 / 3$ & $73 / 2$ & $47 / 0$ & $20 / 0$ & $9 / 0$ & $40 / 0$ \\
\hline 86 & $32 / 0$ & $86 / 3$ & $174 / 5$ & $117 / 4$ & $85 / 2$ & $47 / 2$ & $27 / 1$ & $10 / 0$ & $55 / 1$ \\
\hline 87 & $20 / 0$ & $82 / 1$ & $162 / 8$ & $124 / 5$ & $75 / 4$ & $49 / 2$ & $24 / 0$ & $9 / 0$ & $47 / 0$ \\
\hline 88 & $26 / 0$ & $75 / 4$ & $176 / 7$ & $119 / 6$ & $87 / 4$ & $53 / 1$ & $27 / 1$ & $10 / 0$ & $47 / 2$ \\
\hline 89 & $26 / 0$ & $86 / 3$ & $164 / 10$ & $126 / 7$ & $84 / 3$ & $49 / 2$ & $24 / 0$ & $9 / 0$ & $56 / 0$ \\
\hline 90 & $21 / 1$ & $77 / 6$ & $170 / 14$ & $121 / 8$ & $77 / 6$ & $45 / 2$ & $21 / 1$ & $7 / 0$ & $39 / 3$ \\
\hline & & & & & & & & & \\
\hline
\end{tabular}


4.3. Examples of minimal degenerations. The last tables show, for two modules $U, V$ over an $\tilde{E}_{6}$-quiver, up to symmetry and duality all minimal extensions $M<U \oplus V$ by marking them in the piece of the Auslander-Reiten quiver lying between the two modules. By analyzing such examples we were led to theorem 1 . The interested reader can find the same kind of pictures for all minimal disjoint degenerations at http://wmaz . math. uni-wuppertal.de.
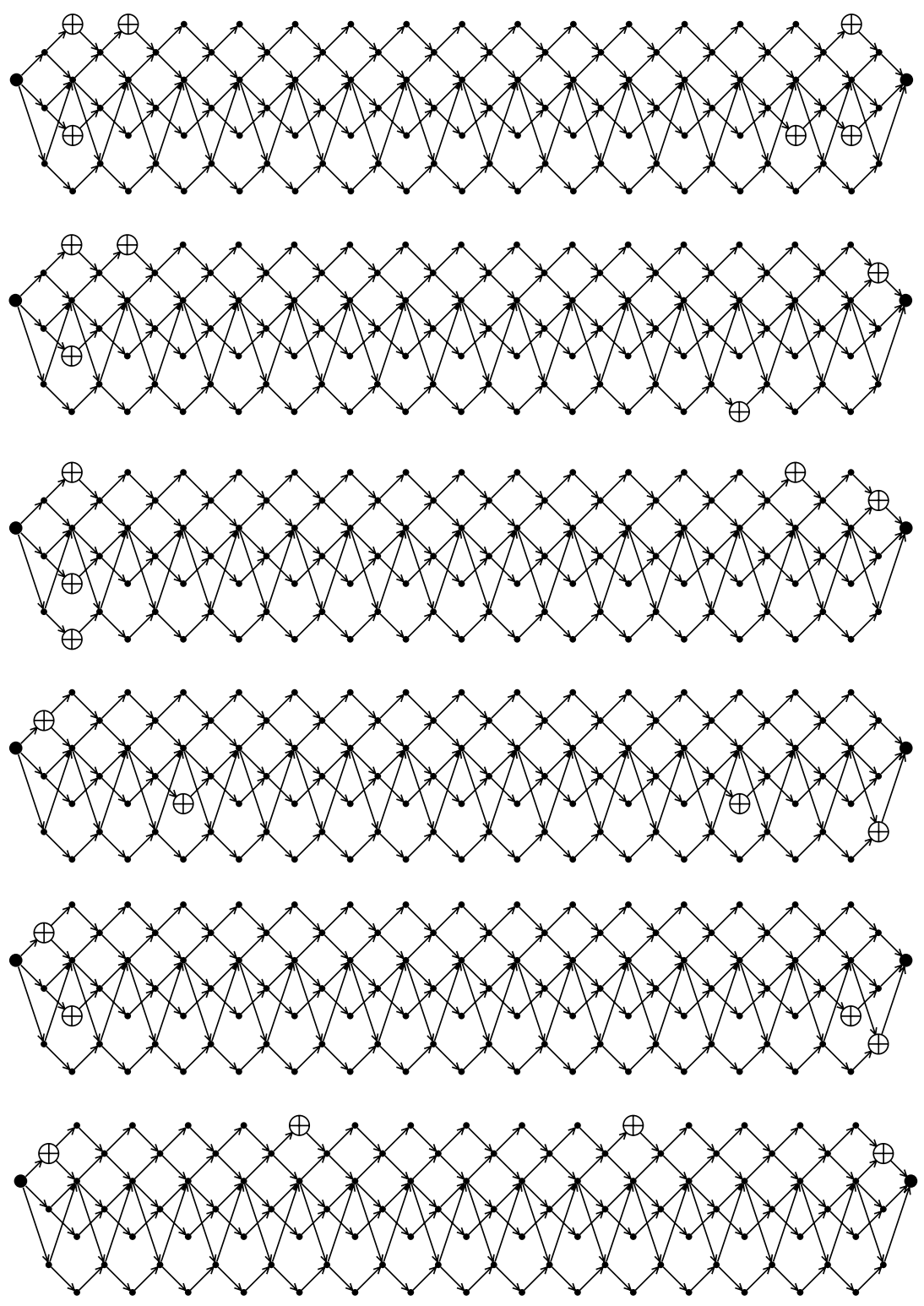


\section{REFERENCES}

[1] Abeasis, S., del Fra, A.: Degenerations for the representations of a quiver of type $A_{m}$, J.Algebra 93 (1985), 376-412. MR 86j:16028

[2] Auslander, M., Reiten, I., Smalø, S.O.: Representation theory of artin algebras, Cambridge Studies in Advanced Mathematics Vol. 3, Cambridge University Press. MR 96c:16015

[3] Bender, J., Bongartz, K.: Minimal singularities in orbits of matrix pencils, to appear in Linear Algebra Appl.

[4] Bongartz, K: On degenerations and extensions of finite dimensional modules, Adv.in Math. 121 (1996), 245-287. MR 98e:16012

[5] Bongartz, K.: Minimal singularities for representations of Dynkin quivers, Comment. Math. Helv. 69(1994), 575-611. MR 96f:16016

[6] Bourbaki, N.: Groupes et algebres de Lie, chapitres 4,5 et 6, Hermann, Paris 1968. MR 39:1590

[7] Dlab, V., Ringel, C.M.: Indecomposable representations of graphs and algebras, Memoirs Amer. Math. Soc., Vol.173, 1976. MR 56:5657

[8] Fritzsche, T.: Minimale disjunkte Entartungen zwischen präprojektiven Darstellungen Euklidischer Kcher, Diplomarbeit BUGH Wuppertal, 2001.

[9] Kac, V.G.: Infinite root systems, representations of graphs and invariant theory, Invent. Math. 56 (1980), 57-92. MR 82j:16050

[10] Markolf, U.: Entartungen von Moduln über darstellungsgerichteten Algebren, Diplomarbeit 1990, Bergische Universität Wuppertal.

[11] Moody, R.V.: Euclidean Lie algebras, Can. J. Math. 21 (1969), 1432-1454. MR 41:287

[12] Riedtmann, C.: Degenerations for representations of quivers with relations, Ann. Sci. Ecole Norm. Sup.(4) 19 (1986), 275-301. MR 88b:16051

[13] Ringel, C.M.: Tame algebras and integral quadratic forms, Lecture Notes in Mathematics 1099, Springer 1984. MR 87f:16027

FB Mathematik BUGH Wuppertal, Gaussstrasse 20, 42119 Wuppertal, Germany

E-mail address: Klaus.Bongartz@math.uni-wuppertal.de

FB Mathematik BUGH Wuppertal, Gaussstrasse 20, 42119 Wuppertal, Germany

E-mail address: tf@noto.de 